\title{
Scalarized black holes in the presence of the coupling to Gauss-Bonnet gravity
}

\author{
Masato Minamitsuji and Taishi Ikeda ${ }^{1}$ \\ ${ }^{1}$ Centro de Astrofísica e Gravitação - CENTRA, \\ Departamento de Física, Instituto Superior Técnico - IST, \\ Universidade de Lisboa - UL, Av. Rovisco Pais 1, 1049-001 Lisboa, Portugal
}

\begin{abstract}
In this paper, we study static and spherically symmetric black hole $(\mathrm{BH})$ solutions in the scalartensor theories with the coupling of the scalar field to the Gauss-Bonnet $(\mathrm{GB})$ term $\xi(\phi) R_{\mathrm{GB}}$, where $R_{\mathrm{GB}}:=R^{2}-4 R^{\alpha \beta} R_{\alpha \beta}+R^{\alpha \beta \mu \nu} R_{\alpha \beta \mu \nu}$ is the GB invariant and $\xi(\phi)$ is a function of the scalar field $\phi$. Recently, it was shown that in these theories scalarized static and spherically symmetric $\mathrm{BH}$ solutions which are different from the Schwarzschild solution and possess the nontrivial profiles of the scalar field can be realized for certain choices of the coupling functions and parameters. These scalarized BH solutions are classified in terms of the number of nodes of the scalar field. It was then pointed out that in the case of the pure quadratic order coupling to the GB term, $\xi(\phi)=\eta \phi^{2} / 8$, scalarized BH solutions with any number of nodes are unstable against the radial perturbation. In order to see how a higher order power of $\phi$ in the coupling function $\xi(\phi)$ affects the properties of the scalarized BHs and their stability, we investigate scalarized $\mathrm{BH}$ solutions in the presence of the quartic order term in the GB coupling function, $\xi(\phi)=\eta \phi^{2}\left(1+\alpha \phi^{2}\right) / 8$. We clarify that the existence of the higher order term in the coupling function can realize scalarized BHs with zero nodes of the scalar field which are stable against the radial perturbation.
\end{abstract}

\section{INTRODUCTION}

\section{A. Black holes in the scalar-tensor theories}

Scalar fields ubiquitously appear in the various contexts of theoretical physics. The Lovelock theorem [1] states that in four dimensions any rank-2, symmetric, and divergence-free tensor $A^{\mu \nu}$, which depends on the metric tensor $g^{\mu \nu}$ and its first order derivatives and linearly on its second order derivatives, is given by a combination of the Einstein tensor $G^{\mu \nu}$ and $g^{\mu \nu}$ itself, $A^{\mu \nu}=p G^{\mu \nu}+q g^{\mu \nu}$ with $p$ and $q$ being constants. The theorem shows that the only diffeomorphism invariant gravitational theory, which is composed of only the metric tensor and its derivatives, is general relativity (GR) with or without a cosmological constant. In other words, any violation of the assumptions in the Lovelock theorem, such as (i) fields other than the metric, (ii) dimensionality of spacetime larger than four, (iii) existence of more than second order derivatives of the metric, and/or (iv) nonlocality, lead to gravitational theories which possess scalar and/or vector field degrees of freedom after suitable mathematical transformations such as dimensional reduction and conformal/disformal transformations, namely scalar-tensor (or vector-tensor) theories $[2]$.

The necessity of scalar fields has arisen not only theoretically but also observationally. The latest issues in cosmology about the existence of primordial inflation and the late-time acceleration of the Universe have motivated us to introduce some new sectors beyond the conventional framework of GR and/or the Standard Model of particle physics. Scalar fields have provided the simplest but most convenient frameworks which could realize accelerated expansion of the early or late-time Universe. On the other hand, the suppression of extra scalar forces for the optimistic choice of parameters requires some screening mechanisms [3], which exist beyond the conventional scheme of the scalartensor theories. Studies in this direction have rediscovered the Horndeski theories [4-7], known as the most general scalar-tensor theories with the second order equations of motion. Moreover, the scalar-tensor theories to explain the accelerated expansion of the Universe have been extended to the beyond Horndeski theories [8-10].

Besides studies of cosmology and phenomenology, physics of black holes (BHs) will also be able to probe the existence of scalar fields from the completely different aspects. In strong gravity regimes in/around BHs and relativistic stars, nonlinearities of the gravitational field equations become most efficient and the deviations from GR will appear most significantly $[2,11]$. One of the criteria for classifying the scalar-tensor theories in terms of BH physics is the existence of the no-hair theorem for asymptotically flat BH solutions. It has been shown that the BH no-hair theorem holds for the scalar-tensor theories with canonical or noncanonical kinetic term, non-negative potential, and nonminimal coupling of the scalar field to the scalar curvature (See e.g., Refs. [12-21].), and the shift symmetric subclass of the (beyond) Horndeski theories with the regular coupling functions of the scalar field $\phi$ and the canonical kinetic term $X:=-(1 / 2) g^{\mu \nu} \partial_{\mu} \phi \partial_{\nu} \phi[22,23]$. For the class of scalar-tensor theories which share the same solutions with GR and satisfy all the conditions for the no-hair theorem, the Schwarzschild and Kerr solutions are the unique solutions of the gravitational field equations in the static and spherically symmetric, and the stationary and axisymmetric cases, respectively. Thus, in order to test the existence of the scalar field degree of freedom, perturbative properties of BHs 
have to be investigated, since the behavior of the perturbations may be different, even if GR and scalar-tensor theories share the same BH solutions [24, 25].

On the other hand, in the scalar-tensor theories, where some of the conditions for the no-hair theorem are violated, BH solutions different from the Schwarzschild and Kerr solutions may exist. Such a BH would be equipped with a nontrivial profile of the scalar field outside the event horizon. One of the scalar-tensor theories which admit BH solutions absent in GR is the scalar-tensor theory with the coupling of the scalar field to the Gauss-Bonnet (GB) term $\xi(\phi) R_{\mathrm{GB}}$ in the Lagrangian density, where $\xi(\phi)$ is an arbitrary regular function of the scalar field, and

$$
R_{\mathrm{GB}}:=R^{2}-4 R^{\alpha \beta} R_{\alpha \beta}+R^{\alpha \beta \mu \nu} R_{\alpha \beta \mu \nu},
$$

is the GB term (See Eq. (2) below.). BH solutions in the scalar-tensor theories with the coupling to the GB term have been studied since 1990s. The first studies have focused on exponential type couplings $\xi(\phi) \propto e^{-c \phi}$ with $c$ being constant, which is motivated by string theory. ${ }^{*} 1$ The static and spherically symmetric BH solutions have been investigated in Refs. [26-35] and then generalized to slowly rotating [36-38] and full rotating [39, 40] solutions accordingly.

The linear coupling of the scalar field to the GB term $\xi(\phi)=c^{\prime} \phi$ with $c^{\prime}$ being constant has also been considered, since it corresponds to a class of the shift-symmetric Horndeski theories with the logarithmic dependence of the coupling functions on $X$, which does not meet one of the conditions for the BH no-hair theorem [22]. Static and spherically symmetric BH solutions in the presence of the linear coupling of the scalar field to the GB term have been explicitly constructed in Refs. [41,42]. They considered the expansion of the equations of motion in terms of the small dimensionless coupling constant $\hat{c}:=c^{\prime} / r_{h}^{2} \ll 1$, where $r_{h}$ is the radial position of the event horizon. At the order of $\hat{c}$, the scalar field is sourced by the GB term of the Schwarzschild spacetime, and at the order of $\hat{c}^{2}$, the deviation from the Schwarzschild geometry appears due to the backreaction of the scalar field. The regularity of the scalar field at the horizon $r=r_{h}$ relates the mass with the scalar charge, and, hence, the scalar charge is not independent of the mass.

It is important to emphasize that if one considers the coupling function $\xi(\phi)$ which is the monotonic function of $\phi$, such as $\xi=e^{c \phi}$ and $\xi=c^{\prime} \phi$, there can be only the nontrivial $\mathrm{BH}$ solutions, while the Schwarzschild and Kerr metrics are not solutions. On the other hand, if the coupling function $\xi(\phi)$ has at least one extremum at $\phi=\phi_{0}, \xi^{(1)}\left(\phi_{0}\right)=0$ where $\xi^{(n)}(\phi):=\partial^{n} \xi(\phi) / \partial \phi^{n}$, there can exist the Schwarzschild and Kerr solutions with $\phi=\phi_{0}$ as a solution, as discussed in Sec. IB.

\section{B. Spontaneous scalarization}

In this paper, we consider the scalar-tensor theory which is composed of the Einstein-Hilbert term, the canonical kinetic term, the coupling of the scalar field to the GB term, and the matter field directly coupled to the scalar field:

$$
S=\frac{1}{2 \kappa^{2}} \int d^{4} x \sqrt{-g}\left(R-\frac{1}{2} g^{\mu \nu} \partial_{\mu} \phi \partial_{\nu} \phi+\xi(\phi) R_{\mathrm{GB}}\right)+\int d^{4} x \sqrt{-\tilde{g}} \mathcal{L}_{m}\left[\tilde{g}_{\mu \nu}, \Psi\right],
$$

where $\kappa^{2}=8 \pi G$ with $G$ being the gravitational constant, the Greek indices $\mu, \nu, \cdots$ run over the space and time of the four-dimensional spacetime, $g_{\mu \nu}$ is the metric tensor, $g=\operatorname{det}\left(g_{\mu \nu}\right)$ is its determinant, $R$ is the scalar curvature associated with $g_{\mu \nu}, \phi$ is the scalar field, and $\mathcal{L}_{m}$ is the Lagrangian for the matter field $\Psi$. The Jordan frame metric $\tilde{g}_{\mu \nu}$ is related to the Einstein frame one by $\tilde{g}_{\mu \nu}=e^{2 A(\phi)} g_{\mu \nu}$, where $A(\phi)$ is the conformal factor depending on the scalar field only. Throughout the paper, we set $c=\hbar=1$.

Varying the action (2) with respect to the metric $g_{\mu \nu}$, the gravitational equation of motion is given by [31-34]

$$
G_{\mu \nu}=\frac{1}{2}\left(\nabla_{\mu} \phi \nabla_{\nu} \phi-\frac{1}{2} g_{\mu \nu} \nabla^{\lambda} \phi \nabla_{\lambda} \phi\right)-4\left(\nabla^{\rho} \nabla^{\sigma} \xi(\phi)\right) P_{\mu \rho \nu \sigma}+\kappa^{2} T_{\mu \nu}
$$

where

$$
P_{\mu \nu \rho \sigma}:=R_{\mu \nu \rho \sigma}+g_{\mu \sigma} R_{\rho \nu}-g_{\mu \rho} R_{\sigma \nu}+g_{\nu \rho} R_{\sigma \mu}-g_{\nu \sigma} R_{\rho \mu}+\frac{R}{2}\left(g_{\mu \rho} g_{\nu \sigma}-g_{\mu \sigma} g_{\nu \rho}\right) .
$$

*1 Although a constant term may be added to the coupling function $\xi(\phi)$, since it does not contribute to the equations of motion in four dimensions, we will not consider it in the rest of the paper. 
The matter energy-momentum tensors in the Jordan and Einstein frames, respectively, are defined by

$$
\tilde{T}_{\mu \nu}:=-\frac{2}{\sqrt{-\tilde{g}}} \frac{\delta\left(\sqrt{-\tilde{g}} \mathcal{L}_{m}\left[\tilde{g}_{\mu \nu}, \Psi\right]\right)}{\delta \tilde{g}^{\mu \nu}}, \quad T_{\mu \nu}:=-\frac{2}{\sqrt{-g}} \frac{\delta\left(\sqrt{-\tilde{g}} \mathcal{L}_{m}\left[\tilde{g}_{\mu \nu}, \Psi\right]\right)}{\delta g^{\mu \nu}},
$$

which are related by

$$
\tilde{T}_{\mu \nu}=\sqrt{\frac{g}{\tilde{g}}} \frac{\delta g^{\alpha \beta}}{\delta \tilde{g}^{\mu \nu}} T_{\alpha \beta}=e^{-2 A(\phi)} T_{\mu \nu} .
$$

On the other hand, varying the action (2) with respect to the scalar field $\phi$, the equation of motion for the scalar field is given by

$$
\square \phi+\xi^{(1)}(\phi) R_{\mathrm{GB}}+2 \kappa^{2} A^{(1)}(\phi) T_{\mu}^{\mu}=0,
$$

where $\square \phi=g^{\mu \nu} \nabla_{\mu} \nabla_{\nu} \phi$ is the d'Alembertian operator and $A^{(n)}(\phi):=\partial^{n} A(\phi) / \partial \phi^{n}$.

In the case that $\xi(\phi)$ and $A(\phi)$ satisfy $\xi^{(1)}\left(\phi_{0}\right)=A^{(1)}\left(\phi_{0}\right)=0$ at $\phi=\phi_{0}=$ constant, where the scalar field equation of motion (7) is trivially satisfied, since $\nabla_{\rho} \nabla_{\sigma} \xi=\partial_{\rho} \partial_{\sigma} \xi-\Gamma_{\rho \sigma}^{\alpha} \partial_{\alpha} \xi=\xi^{(1)} \partial_{\rho} \partial_{\sigma} \phi+\xi^{(2)} \partial_{\rho} \phi \partial_{\sigma} \phi-\Gamma_{\rho \sigma}^{\alpha} \xi^{(1)} \partial_{\alpha} \phi=0$ at $\phi=\phi_{0}$, the gravitational equation of motion (3) reduces to the GR one:

$$
G_{\mu \nu}=\kappa^{2} T_{\mu \nu},
$$

where we assumed that $A\left(\phi_{0}\right)=0$. Thus, the metric $g_{\mu \nu}$ satisfies the Einstein equation (8) and the constant scalar field $\phi=\phi_{0}$, such that $\xi^{(1)}\left(\phi_{0}\right)=A^{(1)}\left(\phi_{0}\right)=0$ is a solution of the theory (2). For instance, if $\xi(\phi) \propto \phi^{2}$ and $A(\phi) \propto \phi^{2}$, a solution in GR with $\phi=0$ is a solution. Nevertheless, this does not guarantee the uniqueness of the GR solution with a constant scalar field and there may be another solution with a nontrivial profile of the scalar field. If the theory (2) admits two or more solutions and if one of them is the GR solution with a constant scalar field, an interesting question is which solution is dynamically favored? If the GR solution is unstable against the linear perturbation in a local region of the spacetime, the so-called spontaneous scalarization may take place.

Spontaneous scalarization has been argued for relativistic stars in the scalar-tensor theory with the coupling of the scalar field to the matter field $A(\phi) \neq 0$ [and without the coupling to the GB term $\xi(\phi)=0$ ], which is triggered by a tachyonic instability of the scalar field due to the coupling to the matter field. In the simplest realization discussed by Damour and Esposito-Farèse [43, 44], the scalarization occurs for the coupling $A(\phi)=\beta \phi^{2} / 8$, where $\beta$ is a constant. The presence of the scalar field can significantly modify the properties of relativistic stars, while satisfying the weak field constraints. Irrespective of the choice of the equation of state, the scalarization occurs for $\beta \lesssim-4.35$ [45, 46], while the binary-pulsar observations have put the bounds $\beta \gtrsim-4.5[47]$.

\section{Gravitational spontaneous scalarization: Test field analysis}

\section{Tachyonic instability of the Schwarzschild solution}

Following the structure of Eq. (7), we expect that spontaneous scalarization may take place even in a vacuum spacetime with $\mathcal{L}_{m}=0$ in Eq. (2). In the case of the spherically symmetric spacetime, a tachyonic instability of the vacuum Schwarzschild solution

$$
d s^{2}=-\left(1-\frac{2 M}{r}\right) d t^{2}+\frac{d r^{2}}{1-2 M / r}+r^{2}\left(d \theta^{2}+\sin ^{2} \theta d \varphi^{2}\right),
$$

could be triggered due to the coupling of the scalar field to the GB term $\xi(\phi) \neq 0$, where $r$ and $M$ are the radial coordinate and mass of the Schwarzschild spacetime, respectively.

The simplest case that the scalar-tensor theory (2) admits the Schwarzschild solution (9) is the case of the quadratic order coupling of the scalar field to the GB term [48]:

$$
\xi(\phi)=\frac{\eta}{8} \phi^{2},
$$

where $\eta(>0)$ is a constant with mass dimension $(-2)$, for which the metric (9) with $\phi=0$ is a solution to the theory (2). In general, the Schwarzschild solution with $\phi=0$ can be realized for a more general coupling function with the higher order powers of the scalar field:

$$
\xi(\phi)=\frac{\eta}{8} \phi^{2}+\sum_{i \geq 3} c_{i} \phi^{i},
$$


where $c_{i}(i=3,4,5, \cdots)$ are constants with mass dimension $(-2)$.

For the coupling (11), we then consider the small perturbation of the scalar field $\delta \phi\left(x^{\mu}\right)$ about the Schwarzschild solution (9) with $\phi=0$. By neglecting the metric perturbations, $\delta \phi\left(x^{\mu}\right)$ obeys the equation

$$
\square_{\mathrm{Sch}} \delta \phi+\xi^{(1)}(\delta \phi) R_{\mathrm{GB}}^{(\mathrm{Sch})}=\left(\square_{\mathrm{Sch}}+\frac{\eta}{4} R_{\mathrm{GB}}^{(\mathrm{Sch})}\right) \delta \phi+\mathcal{O}\left(\delta \phi^{2}\right)=0,
$$

where $R_{\mathrm{GB}}^{(\mathrm{Sch})}=48 M^{2} / r^{6}$ is the GB term for the Schwarzschild solution (9) and $\square_{\mathrm{Sch}}$ is the d'Alembertian operator on the Schwarzschild background (9). Decomposing $\delta \phi\left(x^{\mu}\right)$ into the partial modes:

$$
\delta \phi\left(x^{\mu}\right)=\sum_{\ell, m} e^{-i \omega_{\ell m} t} \frac{\sigma_{\ell m}(r)}{r} Y_{\ell m}(\Omega),
$$

where $Y_{\ell m}(\Omega)$ is the spherical harmonics, from Eq. (12) the radial mode equation is given by

$$
\left[-\frac{d^{2}}{d r_{*}^{2}}+V_{\mathrm{eff}}(r)\right] \sigma_{\ell m}(r)=\omega_{\ell m}^{2} \sigma_{\ell m}(r),
$$

where $d r_{*}:=d r /(1-2 M / r)$ is the tortoise radial coordinate for the Schwarzschild spacetime and the effective potential is given by

$$
V_{\mathrm{eff}}(r)=(r-2 M)\left[\frac{2 M}{r^{7}}\left(r^{3}-6 M \eta\right)+\frac{\ell(\ell+1)}{r^{3}}\right] .
$$

For the radial perturbation $(\ell=0)$, in the vicinity of the horizon $r=2 M$, the effective potential (15) can be approximated by $V_{\text {eff }}(r) \simeq\left(4 M^{2}-3 \eta\right)(r-2 M) /\left(32 M^{5}\right)$, and hence $V_{\text {eff }}(r)$ contains a negative region for $\eta / M^{2}>$ $4 / 3 \approx 1.333$, implying the appearance of pure imaginary modes, i.e., the modes with imaginary $\omega$. On the other hand, for $\eta / M^{2}<4 / 3$ there is no negative region in the effective potential, and, hence, the Schwarzschild solution (9) with $\phi=0$ is expected to be linearly stable for the radial perturbation. From Eq. (15), we also find that, if the effective potential $V_{\text {eff }}(r)$ for the radial perturbation $(\ell=0)$ is non-negative, $V_{\text {eff }}(r)$ for the nonradial perturbation $(\ell \geq 1)$ is also non-negative.

\section{Test scalar field solutions: Pure quadratic coupling}

In order to check the existence of the scalarized $\mathrm{BH}$ solutions in the case without the matter field $\mathcal{L}_{m}=0$ in a semiquantitative way, we present static solutions of the test scalar field by neglecting the backreaction on the spacetime geometry. We will solve the scalar field equation of motion (7) under the static and spherically symmetric ansatz $\phi=\psi(r)$ on a fixed Schwarzschild background (9). For a GB coupling function $\xi(\phi)$, the scalar field equation of motion Eq. (7) reduces to

$$
(r-2 M) \psi^{\prime \prime}(r)+\frac{2(r-M)}{r} \psi^{\prime}(r)+\frac{48 M^{2}}{r^{5}} \xi^{(1)}(\psi)=0,
$$

where a "prime" denotes the derivative with respect to the radial coordinate $r$. The general regular solution of the static scalar field $\phi=\psi(r)$ in the vicinity of the event horizon $r=2 M$ is given by

$$
\psi(r)=\psi_{0}-\frac{3 \xi^{(1)}\left(\psi_{0}\right)}{2 M^{3}}(r-2 M)+\mathcal{O}\left((r-2 M)^{2}\right),
$$

where $\psi_{0}$ is a constant, which represents the value of the scalar field at the event horizon and can take an arbitrary nonzero value, and we have assumed the regularity of the coupling function $\xi(\phi)$. We note that besides Eq. (17) there is the second solution of Eq. (16), which is singular at the horizon and, hence, not considered here. Similarly, the solution for the scalar field at the asymptotic spatial infinity $r \rightarrow \infty$ is given by

$$
\psi(r)=\psi_{\infty}+\frac{Q}{r}+\frac{M Q}{r^{2}}+\frac{4 M^{2} Q}{3 r^{3}}+\frac{2 M^{2}\left(M Q-2 \xi^{(1)}\left(\psi_{\infty}\right)\right)}{r^{4}}+\mathcal{O}\left(\frac{1}{r^{5}}\right),
$$

where $\psi_{\infty}$ and $Q$ are constants, which represent the values of the scalar field in the limit of $r \rightarrow \infty$ and the scalar charge, respectively. 
We numerically solve Eq. (16) from a point sufficiently close to the horizon toward the region of large $r$, under the boundary conditions obtained from Eq. (17):

$$
\psi(r \rightarrow 2 M)=\psi_{0} \neq 0, \quad \psi^{\prime}(r \rightarrow 2 M)=-\frac{3 \xi^{(1)}\left(\psi_{0}\right)}{2 M^{3}} .
$$

We also choose the boundary condition $\psi_{\infty}=0$ at the spatial infinity, as the scalarization of BHs is triggered by a tachyonic instability of the trivial solution $\psi=0$. For $\psi_{0}=0$, there is only the trivial solution $\psi=0$. On the other hand, for $\psi_{0} \neq 0$ there will be a nontrivial scalar field solution $\psi(r) \neq 0$. The scalar charge $Q$ is read from the behavior of the scalar field at the spatial infinity

$$
Q=-\left.r^{2} \psi^{\prime}(r)\right|_{r \rightarrow \infty}
$$

For our numerical analysis, we set $M=1$ and integrate (16) from $r=2 M+10^{-5}$ to $r=1.0 \times 10^{5}$. The scalar charge $Q$ is then numerically evaluated via Eq. (20) at $r=1.0 \times 10^{5}$.

The small perturbation about the static scalar field solution, $\phi=\psi(r)+\delta \phi\left(x^{\mu}\right)$, obeys the equation

$$
\left(\square_{\mathrm{Sch}}-m_{\mathrm{eff}}^{2}\right) \delta \phi=0,
$$

where $m_{\mathrm{eff}}^{2}:=-\xi^{(2)}(\psi) R_{\mathrm{GB}}^{(\mathrm{Sch})}$ is the effective mass squared of the scalar field perturbation. Decomposing $\delta \phi$ as Eq. (13) and deriving the radial mode equation as Eq. (14) with the tortoise coordinate of the Schwarzschild spacetime $r_{*}$, the effective potential for each mode of the linear perturbation $\delta \phi\left(x^{\mu}\right)$ is then given by

$$
V_{\text {eff }}(r)=(r-2 M)\left[\frac{2 M}{r^{7}}\left(r^{3}-24 M \xi^{(2)}(\psi)\right)+\frac{\ell(\ell+1)}{r^{3}}\right] .
$$

For the quadratic order coupling (10) considered in Sec. IC 1, as shown in Fig. 1, the scalar field solutions of Eq. (16) with zero, one and two nodes can be numerically obtained for $\eta / M^{2}=2.90,19.5,50.9$ [48], respectively. ${ }^{*} 2$ The scalar charge $Q$ is given by

$$
\frac{Q}{\sqrt{\eta}}=0.525 \psi_{0},-0.250 \psi_{0}, 0.174 \psi_{0},
$$

for the solutions with zero, one, and two nodes. For the quadratic order coupling to the GB term (10), from Eq. (21)

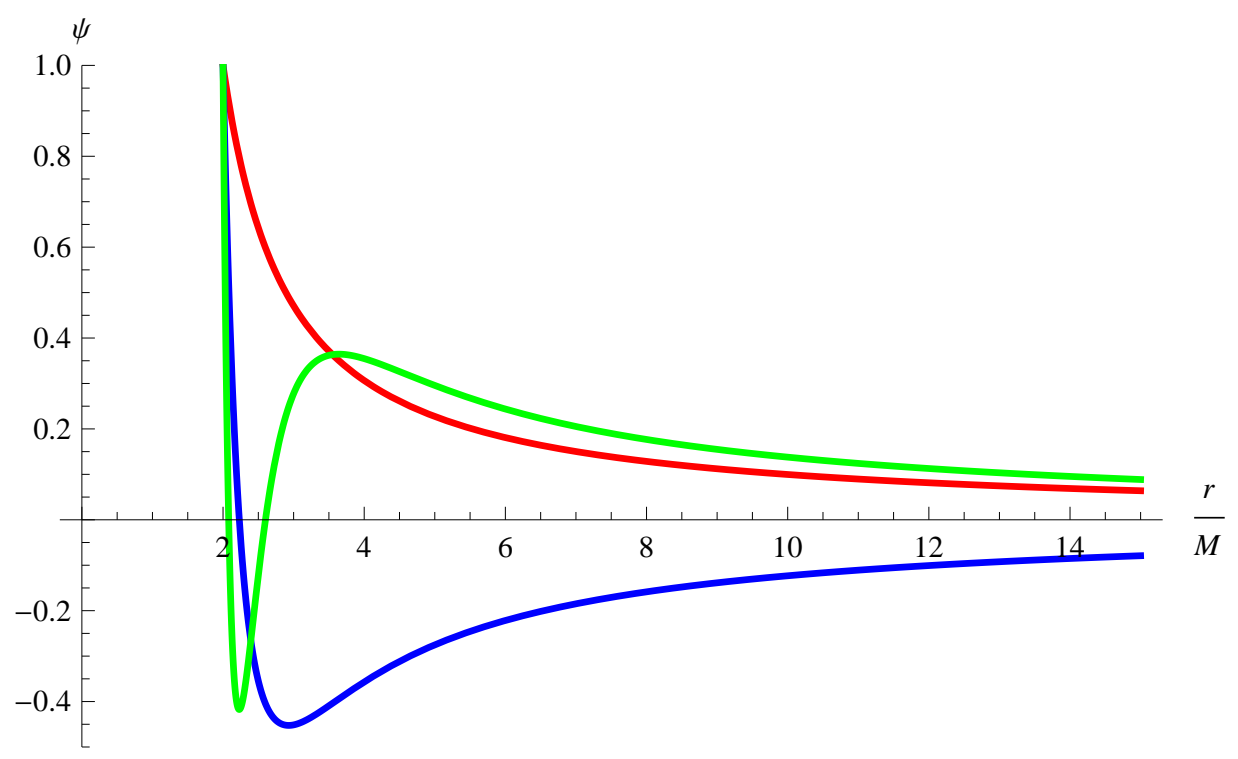

FIG. 1. The solutions $\psi(r)$ to Eq. (16) for the quadratic order coupling to the GB term (10) with the boundary conditions $\psi_{0}=1.0$ and $\psi_{\infty}=0$ are shown as the function of $r / M$. The red, blue, and green curves correspond to the scalar field solutions with zero, one, and two nodes of the scalar field, respectively.

*2 There will be solutions of the scalar field with more nodes, but in this paper we focus on the scalar field solutions with zero, one, and two nodes. 
we find that the equation for the small perturbation $\phi=\psi(r)+\delta \phi\left(x^{\mu}\right)$ follows the same equation as the leading order part of Eq. (12). Since all the values $\eta / M^{2}=2.90,19.5,50.9$, for the nontrivial solutions of the scalar field are larger than $4 / 3$, the effective potential (15) for the radial perturbation $(\ell=0)$ contains a negative region and would possess pure imaginary modes. Thus, we expect that the nontrivial scalar field solutions for Eq. (10) are unstable against the radial perturbation.

By taking the backreaction on the spacetime geometry, the explicit solutions of scalarized BH solutions were firstly presented in Refs. [40, 48-51], and then followed by Refs. [52-54]. In Refs. [48] and [49], the scalarized BH solutions for the coupling functions $(10)$ and $\xi(\phi)=\eta\left(1-e^{-3 \phi^{2} / 2}\right) / 12$ in our conventions were investigated, respectively. Ref. [53] analyzed the radial perturbation of the scalarized BH solutions for both the coupling functions. They confirmed the existence of pure imaginary modes for all the scalarized BHs for the quadratic order coupling (10). This instability can be qualitatively understood from our test field analysis shown above. On the other hand, for the coupling function $\xi(\phi)=\eta\left(1-e^{-3 \phi^{2} / 2}\right) / 12$, Ref. [53] also showed that the effective potential for scalarized BH solutions with zero nodes whose mass is greater than the critical mass contains no pure imaginary mode, and, hence, these solutions are radially stable. These results indicate the importance of the existence of the higher order powers of $\phi$ in the coupling constant $\xi(\phi)$ to realize scalarized BH solutions which are stable against the radial perturbation. In order to clarify this point, we will study the coupling function $\xi(\phi)$ which contains the $\phi^{4}$ term as well as the $\phi^{2}$ term.

\section{Test scalar field solutions: General coupling}

In this paper, we will consider the GB coupling function:

$$
\xi(\phi)=\frac{\eta}{8}\left(\phi^{2}+\alpha \phi^{4}\right),
$$

which preserves the symmetry $\xi(-\phi)=\xi(\phi)$, where $\alpha$ is the dimensionless coupling constant. For the coupling function (24), Eq. (16) can be rewritten by

$$
\psi^{\prime \prime}(r)+\frac{2(r-M)}{r(r-2 M)} \psi^{\prime}(r)+\frac{12 \eta M^{2}}{r^{5}(r-2 M)} \psi(r)\left(1+2 \alpha \psi(r)^{2}\right)=0 .
$$

There are several solutions of a constant scalar field: For $\alpha>0, \psi=0$ is only the constant scalar field solution, while for $\alpha<0$, in addition to $\psi=0$, there are two additional constant scalar field solutions

$$
\psi(r)= \pm \sqrt{-\frac{1}{2 \alpha}} .
$$

We note that even if the backreaction is taken into consideration the Schwarzschild metric with these constant values of the scalar field are the solutions of the theory (2). Since $R_{\mathrm{GB}}^{(\mathrm{Sch})}=48 M^{2} / r^{6}>0$ and $\xi^{(2)}( \pm \sqrt{-1 /(2 \alpha)})=-\eta / 2<0$, the effective mass squared for the perturbation $m_{\text {eff }}^{2}=-\xi^{(2)}(\psi) R_{\mathrm{GB}}^{(S c h)}>0$ [See Eq. (21).]; the solution (26) is stable and no spontaneous scalarization takes place. On the other hand, for the solution with $\psi=0, \xi^{(2)}(0)=\eta / 4>0$, and, hence, $m_{\mathrm{eff}}^{2}=-\xi^{(2)}(0) R_{\mathrm{GB}}<0$, indicating the occurrence of a tachyonic instability. In the limit of Eq. (25) to the event horizon $r \rightarrow 2 M$, assuming that the second order derivative $\psi^{\prime \prime}(r)$ is regular, the boundary condition at the horizon can be obtained as

$$
\psi^{\prime}(r=2 M)=-\frac{3 \eta \psi_{0}}{8 M^{3}}\left(1+2 \alpha \psi_{0}^{2}\right) .
$$

Thus, for $\psi_{0}>0$, the decreasing $\psi$ in the vicinity of the horizon imposes $\psi^{\prime}(r=2 M)<0$, and hence

$$
\alpha \psi_{0}^{2}>-\frac{1}{2} .
$$

For $\psi_{0}<0$, we impose $\psi^{\prime}(r=2 M)>0$, leading to the same condition as Eq. (28). For $\psi_{0}= \pm \sqrt{-1 /(2 \alpha)}$ with $\alpha<0$, the solution is given by Eq. (26), which does not satisfy $\psi_{\infty}=0$. Without loss of generality, we assume $\psi_{0}>0$. In Fig. 2, the absolute value of the scalar charge $|Q|$ divided by $\sqrt{\eta}$ is shown as the function of the mass $M$ divided by $\sqrt{\eta}$ for $\psi_{0}=1.0$. We note that $Q>0$ for the scalar field solutions with even number nodes, and $Q<0$ for those with odd number nodes. The red, blue, and green curves correspond to the scalar field solutions with zero, one, and two nodes, respectively. The dashed lines from the bottom correspond to the scalar field solutions with zero, one, and two nodes for the pure quartic order coupling:

$$
\xi(\phi)=\frac{\lambda}{8} \phi^{4}
$$


which are given by $Q / M=0.650 \psi_{0},-0.824 \psi_{0}, 0.943 \psi_{0}$, respectively, where $\lambda$ is the coupling constant with mass dimension $(-2)$. As we will see later, the essential features remain the same even if the backreaction and the metric

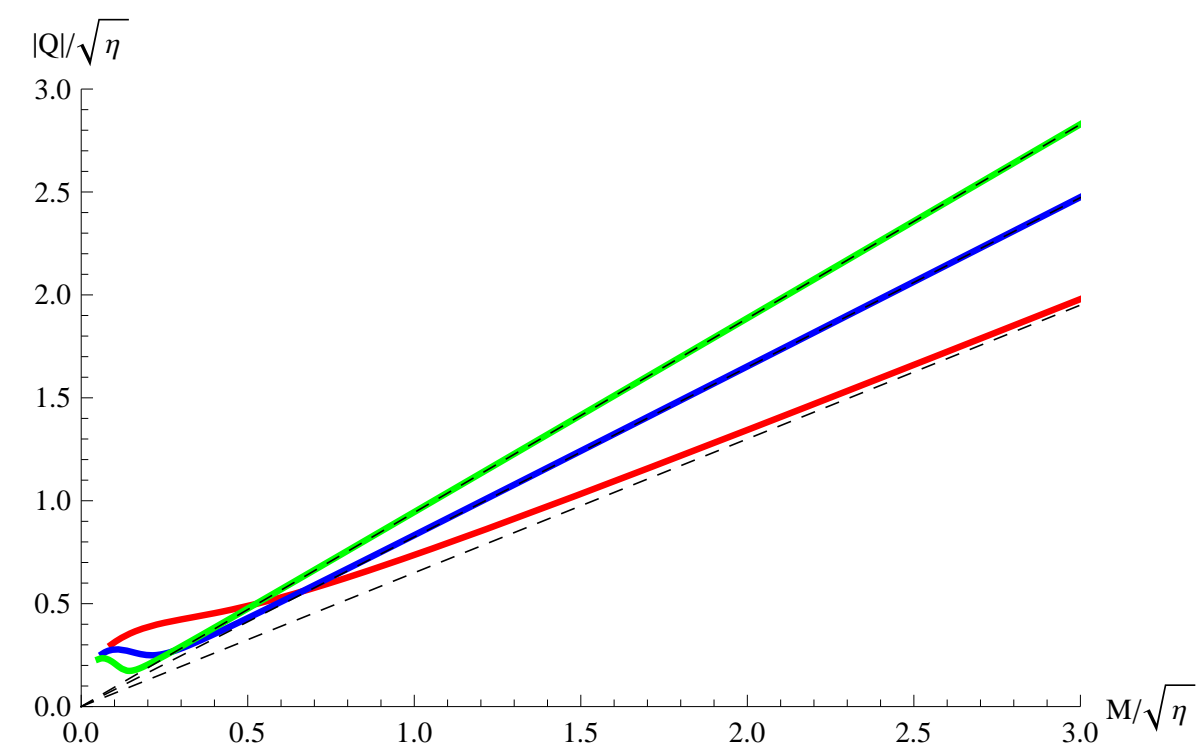

FIG. 2. The absolute value of the scalar charge $|Q|$ divided by $\sqrt{\eta}$ is shown as the function of the mass $M$ divided by $\sqrt{\eta}$ for $\psi_{0}=1.0$. We note that $Q>0$ for the scalar field solutions with an even number of nodes, and $Q<0$ for those with an odd number of nodes. The red, blue, and green curves correspond to the scalar field solutions with zero, one, and two nodes, respectively. The dashed lines from the bottom correspond to the solutions with zero, one, and two nodes for the pure quartic order coupling $\xi(\phi)=\lambda \phi^{4} / 8$, respectively. For $\alpha<0$, we have chosen the minimal value of $\alpha$ to $-0.5+10^{-7}$.

perturbation are included in our analysis.

From Eq. (22), for the coupling (24), the effective potential for each mode of the perturbation $\phi=\psi(r)+\delta \phi\left(x^{\mu}\right)$ is given by

$$
V_{\mathrm{eff}}(r)=(r-2 M)\left[\frac{2 M}{r^{7}}\left(r^{3}-6 \eta M\left(1+6 \alpha \psi^{2}\right)\right)+\frac{\ell(\ell+1)}{r^{3}}\right] .
$$

In Fig. 3, the effective potential $V_{\text {eff }}(r)$ multiplied by $M^{2}$ for the radial perturbation $(\ell=0)$ is shown as the function of $r / M$ for $\psi_{0}=1.0$ for the scalar field solutions with zero, one, and two nodes, respectively. In the top panel, the red, blue, magenta, and green curves correspond to the scalar field solutions with zero nodes for $\left(\eta / M^{2}, \alpha\right)=(2.14,0.30)$, $(2.90,0),(3.32,-0.10),(3.402,-0.1155),(3.59,-0.15)$, respectively. For $\alpha \psi_{0}^{2}=-0.1155$, we find that the local minimum of $V_{\text {eff }}(r)$ for the scalar field solution with zero nodes becomes zero, and, hence, for

$$
-\frac{1}{2}<\alpha \psi_{0}^{2}<-0.1155
$$

$V_{\text {eff }}(r)$ has no negative region. Thus, for such a solution, the spectrum does not contain any pure imaginary mode, and the solution is expected to be stable against the radial perturbation $(\ell=0)$. From Eq. (30), for $\alpha$ satisfying Eq. (31) the solutions of the static scalar field with zero nodes are also expected to be stable against the nonradial perturbations $(\ell \geq 1)$.

Similarly, in the bottom-left panel of Fig. 3, the red, blue, and green curves correspond to the scalar field solutions with one node for $\left(\eta / M^{2}, \alpha\right)=(19.5,0),(31.6,-0.40),(53.5,-0.49)$, and in the bottom-right panel of Fig. 3, the red, blue, and green curves correspond to the scalar field solutions with two nodes for $\left(\eta / M^{2}, \alpha\right)=(50.9,0),(66.4,-0.35)$, $(105.9,-0.49)$, respectively. For the scalar field solutions with one and two nodes, even for a large negative value of $\alpha$, the effective potential always contains a negative region, and hence should contain pure imaginary modes. Thus, these solutions are expected to remain unstable against the radial perturbation. We note that for the constant scalar field solution Eq. (26) the effective potential (30) for the radial perturbation $(\ell=0)$ reduces to

$$
V_{\mathrm{eff}}(r)=\frac{2 M}{r^{7}}(r-2 M)\left(r^{3}+12 \eta M\right)>0,
$$



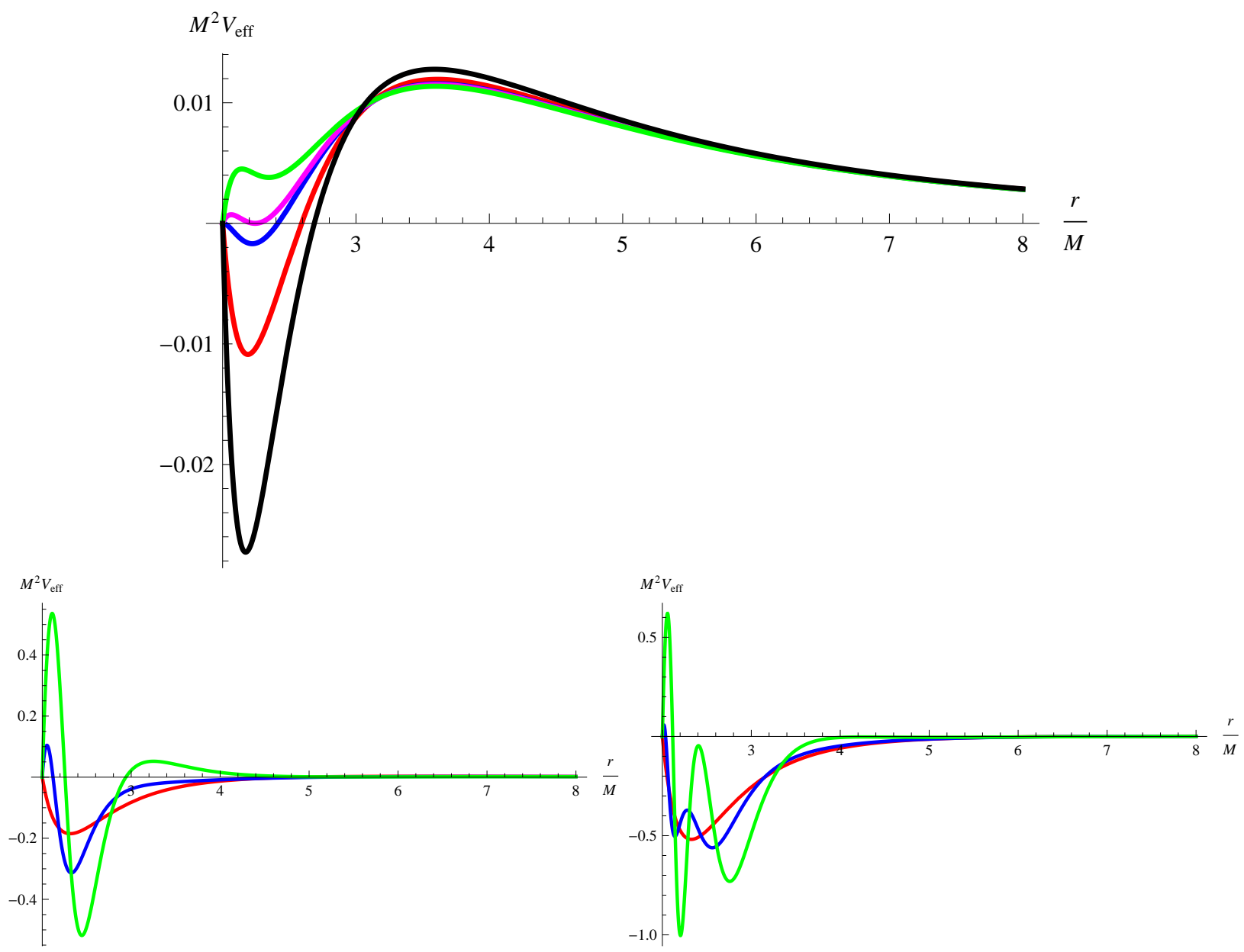

FIG. 3. For the coupling (24), the effective potential $V_{\text {eff }}(r)$ multiplied by $M^{2}$ for the radial perturbation $(\ell=0)$ is shown as the function of $r / M$ for $\psi_{0}=1.0$ for the scalar field solutions with zero, one, and two nodes, respectively. In the top panel, the black, red, blue, magenta, and green curves correspond to the scalar field solutions with zero nodes for $\left(\eta / M^{2}, \alpha\right)=(2.14,0.30)$, $(2.90,0),(3.32,-0.10),(3.402,-0.115),(3.59,-0.15)$, respectively. Similarly, in the bottom-left panel, the red, blue, and green curves correspond to the scalar field solutions with one node for $\left(\eta / M^{2}, \alpha\right)=(19.5,0),(31.6,-0.40),(53.5,-0.49)$, and in the bottom-right panel, the red, blue, and green curves correspond to the scalar field solutions with two nodes for $\left(\eta / M^{2}, \alpha\right)=(50.9,0),(66.4,-0.35),(105.9,-0.49)$, respectively.

which corresponds to the stable Schwarzschild solutions with a constant scalar field. For the nonradial perturbations $(\ell \geq 1)$, we also obtain $V_{\text {eff }}(r)>0$, which confirms the stability of the solution (26).

Since the analysis in this subsection does not include the backreaction on the spacetime geometry and the metric perturbation, the conclusion obtained from the above analysis may change if they are fully taken into consideration. Nevertheless, it is very suggestive that the test field analysis contains the stable scalar field solution with zero nodes if there is a higher power of the scalar field in the coupling function $\xi(\phi)$. This motivates us to construct the scalarized $\mathrm{BH}$ solutions in the scalar-tensor theories (2) by solving the gravitational equation of motion (3) and the scalar field equation of motion (7), and investigate their properties. We will explore these issues in the rest of the paper.

\section{Construction of the paper}

We will organize the rest of the paper as follows: In Sec. II, we will present the strategy for constructing the static and spherically symmetric vacuum BH solutions in the scalar-tensor theories (2). In Sec. III, we will show the explicit solutions of the scalarized BH solutions for the coupling functions (10) and (24). In Sec. IV, we will investigate the stability of the scalarized BH solutions constructed in Sec. III against the radial perturbation. In Sec. V, we will close the paper after giving a brief summary and conclusion. 


\section{STRATEGY}

In the rest of the paper, we will focus on the scalar-tensor theories $(2)$ without the matter field $\mathcal{L}_{m}=0$.

\section{A. Equations of motion}

We assume that the metric is given by the form of a static and spherically symmetric spacetime:

$$
d s^{2}=-A(r) d t^{2}+\frac{d r^{2}}{B(r)}+r^{2}\left(d \theta^{2}+\sin ^{2} \theta d \varphi^{2}\right),
$$

where $A(r)$ and $B(r)$ are the functions of the radial coordinate $r$, and the scalar field $\phi$ is also the function of $r$ :

$$
\phi=\psi(r) .
$$

The $(t, t)$ - and $(r, r)$ - components of the gravitational equation of motion (3) are then given by

$$
\begin{aligned}
& 2\left(r+2(1-3 B) \xi^{(1)} \psi^{\prime}\right) B^{\prime}+\frac{1}{2}\left(-4+B\left(4+r^{2} \psi^{\prime 2}\right)\right)-8 B(B-1)\left(\psi^{\prime 2} \xi^{(2)}+\psi^{\prime \prime} \xi^{(1)}\right)=0, \\
& 2\left(r+2(1-3 B) \xi^{(1)} \psi^{\prime}\right) A^{\prime}-\frac{A}{2 B}\left(4+B\left(-4+r^{2} \psi^{\prime 2}\right)\right)=0 .
\end{aligned}
$$

Similarly, the scalar field equation of motion (7) is given by

$$
\psi^{\prime \prime}+\frac{1}{2}\left(\frac{4}{r}+\frac{A^{\prime}}{A}+\frac{B^{\prime}}{B}\right) \psi^{\prime}+\frac{4}{r^{2} A}(-1+B) \xi^{(1)} A^{\prime \prime}(r)-\frac{2 A^{\prime} \xi^{(1)}}{r^{2} A^{2} B}\left(B^{2} A^{\prime}+A B^{\prime}-A^{\prime} B-3 A B B^{\prime}\right)=0 .
$$

Solving Eq. (36) with respect to $B^{-1}$, we obtain

$$
\frac{1}{B}=\frac{1}{8 A}\left(4 A^{\prime}\left(r+2 \xi^{(1)} \psi^{\prime}\right)+A\left(4-r^{2} \psi^{\prime 2}\right) \pm \sqrt{-384 A A^{\prime} \xi^{(1)} \psi^{\prime}+\left(4 A^{\prime}\left(r+2 \xi^{(1)} \psi^{\prime}\right)+A\left(4-r^{2} \psi^{\prime 2}\right)\right)^{2}}\right) .
$$

For a $\mathrm{BH}$ solution, we require that for $r>r_{h}$, where $r_{h}$ is the position of the event (outermost) horizon, $A>0$, $A^{\prime}>0, B>0, B^{\prime}>0$, and in the limit of $r \rightarrow r_{h}, A \rightarrow 0, B \rightarrow 0, A / B \rightarrow$ constant, and $\psi$ and $\psi^{\prime}$ are regular. Then, in the limit of $r \rightarrow r_{h}$, Eq. (36) can be approximated by

$$
\left.\frac{1}{B} \rightarrow \frac{A^{\prime}}{A}\left(r+2 \xi^{(1)} \psi^{\prime}\right)\right|_{r \rightarrow r_{h}}
$$

which can be obtained only from the (+)-branch of Eq. (38). Thus, in the rest of the paper we only focus on the (+)-branch.

Substituting Eq. (38) into Eqs. (35) and (37), we obtain a set of the equations which are quasilinear for $A^{\prime \prime}(r)$ and $\psi^{\prime \prime}(r)$, and, by arranging them, we obtain the evolution equations for $A(r)$ and $\psi(r)$ with respect to $r$ :

$$
\begin{aligned}
A^{\prime \prime}(r) & =F_{A}\left[A, A^{\prime}, \psi, \psi^{\prime} ; r\right], \\
\psi^{\prime \prime}(r) & =F_{\psi}\left[A, A^{\prime}, \psi, \psi^{\prime} ; r\right],
\end{aligned}
$$

where $F_{A}$ and $F_{\psi}$ are the nonlinear combinations of the given variables which we omit to show explicitly, since they are quite involved. After integrating Eqs. (40) and (41) for $A(r)$ and $\psi(r)$ and substituting them into Eq. (38), $B(r)$ can be obtained.

We also define the right-hand side of the gravitational equation of motion (3) as the effective energy-momentum tensor of the scalar field:

$$
T_{\mu \nu}^{(\mathrm{eff})}:=\frac{1}{2}\left(\nabla_{\mu} \phi \nabla_{\nu} \phi-\frac{1}{2} g_{\mu \nu} \nabla^{\lambda} \phi \nabla_{\lambda} \phi\right)-4\left(\nabla^{\rho} \nabla^{\sigma} \xi(\phi)\right) P_{\mu \rho \nu \sigma}
$$

In the static and spherically symmetric spacetime (33), the effective energy density, the radial pressure, and the tangential pressure, defined by $\rho^{(\mathrm{eff})}(r):=-T^{(\mathrm{eff}) t}{ }_{t}, p_{r}^{(\mathrm{eff})}(r):=T^{(\mathrm{eff}) r}{ }_{r}$, and $p_{t}^{(\mathrm{eff})}(r):=T^{(\mathrm{eff}) \theta_{\theta}}\left[=T^{(\mathrm{eff}) \varphi}{ }_{\varphi}\right]$, respectively, 
are explicitly given by

$$
\begin{aligned}
& \rho^{(\mathrm{eff})}(r)=\frac{1}{4 r^{2}}\left\{8(1-3 B) B^{\prime} \xi^{(1)} \psi^{\prime}+B\left[\psi^{\prime 2} r^{2}-16(B-1)\left(\psi^{\prime 2} \xi^{(2)}+\psi^{\prime \prime} \xi^{(1)}\right)\right]\right\}, \\
& p_{r}^{(\mathrm{eff})}(r)=\frac{B \psi^{\prime}}{4}\left[\frac{8(3 B-1) A^{\prime} \xi^{(1)}}{r^{2} A}+\psi^{\prime}\right], \\
& p_{t}^{(\mathrm{eff})}(r)=\frac{B}{4 r A^{2}}\left\{A \psi^{\prime}\left(12 A^{\prime} B^{\prime} \xi^{(1)}-r A \psi^{\prime}\right)+B\left(-4 A^{\prime 2} \xi^{(1)} \psi^{\prime}+8 A \xi^{(1)} \psi^{\prime} A^{\prime \prime}+8 A A^{\prime}\left(\psi^{\prime 2} \xi^{(2)}+\psi^{\prime \prime} \xi^{(1)}\right)\right)\right\} .
\end{aligned}
$$

\section{B. Boundary conditions, mass, and scalar charge}

The boundary conditions to integrate Eqs. (40) and (41) numerically from (the vicinity of) the horizon can be fixed by solving Eqs. (40) and (41) in the vicinity of the horizon $r=r_{h}$ :

$$
\begin{aligned}
& A=a_{1}\left(r-r_{h}\right)+\mathcal{O}\left(\left(r-r_{h}\right)^{2}\right), \\
& B=\frac{r_{h}\left(r_{h}^{2}-\sqrt{r_{h}^{4}-96 \xi^{(1)}\left(\psi_{0}\right)^{2}}\right)}{48 \xi^{(1)}\left(\psi_{0}\right)^{2}}\left(r-r_{h}\right)+\mathcal{O}\left(\left(r-r_{h}\right)^{2}\right), \\
& \psi=\psi_{0}-\left(\frac{r_{h}^{2}-\sqrt{r_{h}^{4}-96 \xi^{(1)}\left(\psi_{0}\right)^{2}}}{4 r_{h} \xi^{(1)}\left(\psi_{0}\right)}\right)\left(r-r_{h}\right)+\mathcal{O}\left(\left(r-r_{h}\right)^{2}\right),
\end{aligned}
$$

where $\psi_{0}:=\psi\left(r=r_{h}\right)$ is the amplitude of the scalar field at the horizon, $a_{1}$ is an arbitrary constant, and we choose the branch that recovers the Schwarzschild solution in the limit of $\xi^{(1)}\left(\psi_{0}\right) \rightarrow 0$. The condition for the existence of the scalarized $\mathrm{BH}$ solutions is given by

$$
r_{h}^{4}>96 \xi^{(1)}\left(\psi_{0}\right)^{2}
$$

Here, without loss of generality we set

$$
a_{1}=\frac{r_{h}\left(r_{h}^{2}-\sqrt{r_{h}^{4}-96 \xi^{(1)}\left(\psi_{0}\right)^{2}}\right)}{48 \xi^{(1)}\left(\psi_{0}\right)^{2}} .
$$

After integrating Eqs. (40) and (41) to a sufficiently large radius, $r \gg r_{h}$, the solutions approach the asymptotic form which can be obtained by solving Eqs. (40) and (41) in the limit of $r \rightarrow \infty$ :

$$
\begin{aligned}
& A=1-\frac{2 M}{r}+\frac{M Q^{2}}{12 r^{3}}+\frac{M Q}{6 r^{4}}\left(M Q+24 \xi^{(1)}\left(\psi_{\infty}\right)\right)+\mathcal{O}\left(\frac{1}{r^{5}}\right) \\
& B=1-\frac{2 M}{r}+\frac{Q^{2}}{4 r^{2}}+\frac{M Q^{2}}{4 r^{3}}+\frac{M Q}{3 r^{4}}\left(M Q+24 \xi^{(1)}\left(\psi_{\infty}\right)\right)+\mathcal{O}\left(\frac{1}{r^{5}}\right), \\
& \psi=\psi_{\infty}+\frac{Q}{r}+\frac{M Q}{r^{2}}+\frac{1}{r^{3}}\left(\frac{4 M^{2} Q}{3}-\frac{Q^{3}}{24}\right)+\frac{M}{6 r^{4}}\left(12 M^{2} Q-Q^{3}-24 M \xi^{(1)}\left(\psi_{\infty}\right)\right)+\mathcal{O}\left(\frac{1}{r^{5}}\right),
\end{aligned}
$$

where $\psi_{\infty}:=\psi(r \rightarrow \infty)$ is the asymptotic value of the scalar field, and $M$ and $Q$ are the mass and the scalar charge, respectively. From Eq. (47), $M$ and $Q$ can be evaluated as

$$
\begin{gathered}
M=\left.\frac{r}{2}(1-B)\right|_{r \rightarrow \infty}, \\
Q=-\left.r^{2} \psi^{\prime}(r)\right|_{r \rightarrow \infty} .
\end{gathered}
$$

We note that because of the time reparametrization symmetry there is always the degree of freedom to rescale $A$ by some constant factor in Eqs. (44) and (47). We emphasize that the physical quantities such as $M$ and $Q$ are not affected by the rescaling of $A$ at all.

For $\psi_{0}$ such that

$$
\xi^{(1)}\left(\psi_{0}\right)=0
$$


the Schwarzschild solution $A(r)=B(r)=1-2 M / r$ with a constant scalar field $\psi(r)=\psi_{0}$ is always a solution of the theory (2) with $Q=0$.

On the other hand, for $\psi_{0}$ such that $\xi^{(1)}\left(\psi_{0}\right) \neq 0, \psi(r)$ varies with $r$ and hence $Q$ does not vanish in general. After solving Eqs. (40) and (41) for $A(r)$ and $\psi(r)$ iteratively for sufficiently many different sets of the parameters, $\psi_{0}$ and the coupling constants in $\xi(\phi)$, we will find sets of them that satisfy the boundary condition $\psi_{\infty}=0$ in the large distance limit. For $\psi_{0}>0$, from Eq. (44) the decreasing $\psi(r)$ in the vicinity of $r=r_{h}$ requires $\xi^{(1)}\left(\psi_{0}\right)>0$. Similarly, for $\psi_{0}<0$, from Eq. (44) the increasing $\psi(r)$ in the vicinity of $r=r_{h}$ requires $\xi^{(1)}\left(\psi_{0}\right)<0$. Assuming that $\xi(\phi)$ is an even function across $\phi=0$, such as Eqs. (10) and (24), without loss of generality we focus on the case of

$$
\psi_{0}>0, \quad \xi^{(1)}\left(\psi_{0}\right)>0
$$

\section{SCALARIZED BLACK HOLES}

In this section, we present our numerical scalarized BH solutions for several choices of the coupling function $\xi(\phi)$. In order to compare with the analysis in Sec. I C, we will consider the pure quadratic coupling (10) and the quartic order coupling (24), and investigate whether the inclusion of the gravitational backreaction modifies the properties of the solutions. As mentioned in Sec. I C, the first model has been originally investigated in Refs. [48, 53], and we will also confirm the consistency with them.

For the couplings (10) and (24), in order to find the scalarized BH solutions, we will vary the dimensionless parameters $\left(\eta / r_{h}^{2}, \psi_{0}\right)$ and $\left(\eta / r_{h}^{2}, \alpha, \psi_{0}\right)$, respectively. In our numerical analysis, without loss of generality we will fix $r_{h}=1$ and integrate the equations (40) and (41) from $r_{s}=r_{h}+10^{-5}$ with the boundary conditions (44) to $r=5.0 \times 10^{4}$. For such solutions, following Eqs. (48) and (49), $M$ and $Q$ are numerically evaluated at $r=5.0 \times 10^{4}$.

\section{A. Quadratic coupling}

First, we review the case of the quadratic order coupling (10). The condition (45) is given by

$$
r_{h}^{4}>6 \eta^{2} \psi_{0}^{2}
$$

For the quadratic order coupling (10), the condition (51) is automatically satisfied.

In Fig. 4, the absolute value of the scalar charge $|Q|$ divided by $\sqrt{\eta}$ is shown as the function of the mass $M$ divided by $\sqrt{\eta}$. In the left larger panel, the red, blue, and green curves correspond to the scalarized BH solutions with zero, one, and two nodes of the scalar field, respectively. The right smaller panel corresponds to the enlarged display for the scalarized BH solutions with one and two nodes. Although there are solutions with more than three nodes of the scalar field [53], we will not show them explicitly, because of the similarity to the solutions with fewer numbers of nodes. Because of the symmetry $\xi(-\phi)=\xi(\phi)$, if $\left(g_{\mu \nu}, \phi\right)$ is a solution then $\left(g_{\mu \nu},-\phi\right)$ is also a solution. Thus, a scalarized $\mathrm{BH}$ solution with $(M, Q)$ is physically equivalent to that with $(M,-Q)$. For $\psi_{0}>0$, $Q>0$ for the scalarized $\mathrm{BH}$ solutions with an even number of nodes, and $Q<0$ for those with an odd number of nodes.

From Fig. 4, we find that the branch of the scalarized BH solutions with zero nodes bifurcates from the Schwarzschild solution, i.e., the axis of $Q=0$, at $M / \sqrt{\eta}=0.587\left(\eta / M^{2} \approx 2.90\right)$ and extends to $(M / \sqrt{\eta},|Q| / \sqrt{\eta})=(0.636,0.275)$, where the bound (52) is saturated. The bifurcation point agrees with the case of the test field analysis. For all the cases of scalarized $\mathrm{BH}$ solutions, as the value of $\psi_{0}$ increases, the value of $|Q|$ also increases. The difference because of the inclusion of the backreaction is observed as the varying $M / \sqrt{\eta}$ and the existence of the upper bound on $|Q|$ resulting from the upper bound on $\psi_{0}$. Similarly, the scalarized BH solutions with one and two nodes bifurcate from the Schwarzschild solutions at $M / \sqrt{\eta} \approx 0.226\left(\eta / M^{2} \approx 19.5\right)$ and at $M / \sqrt{\eta} \approx 0.140\left(\eta / M^{2} \approx 50.9\right)$, and extend to $(M / \sqrt{\eta},|Q| / \sqrt{\eta})=(0.233,0.0162)$ and $(M / \sqrt{\eta},|Q| / \sqrt{\eta})=(0.144,0.00464)$, respectively, where the maximal charges are obtained when Eq. (52) is again saturated. These results are consistent with those in Ref. [48, 53].

In Fig. 5, the effective energy density $\rho^{(\mathrm{eff})}(r)$, the effective radial pressure $p_{r}^{(\mathrm{eff})}(r)$, and the effective tangential pressure $p_{t}^{(\text {eff })}(r)$ defined in Eq. (43), which are all multiplied by $r_{h}^{2}$, are shown as the functions of $r / r_{h}$ for $\psi_{0}=0.01$. The top panel corresponds to the cases of the scalarized BH solutions with zero nodes of the scalar field, while the bottom-left and bottom-right panels correspond to the cases of the scalarized BH solutions with one and two nodes of the scalar field, respectively. In each panel, the red, blue, and green curves correspond to $\rho^{(\mathrm{eff})}(r), p_{r}^{(\mathrm{eff})}(r)$, and $p_{t}^{(\text {eff })}(r)$, respectively. For all the solutions, in the vicinity of the horizon $r=r_{h}, \rho^{(\text {eff })}(r)<0, p_{r}^{(\text {eff })}(r)>0$, and $p_{t}^{\text {(eff) }}(r)<0$. On the other hand, irrespective of the number of nodes, for a sufficiently large radius, we always obtain $\rho^{(\mathrm{eff})}(r)>0$ and $p_{r}^{(\mathrm{eff})}(r)>0$, while $p_{t}^{(\mathrm{eff})}(r)<0$ after several oscillations. 


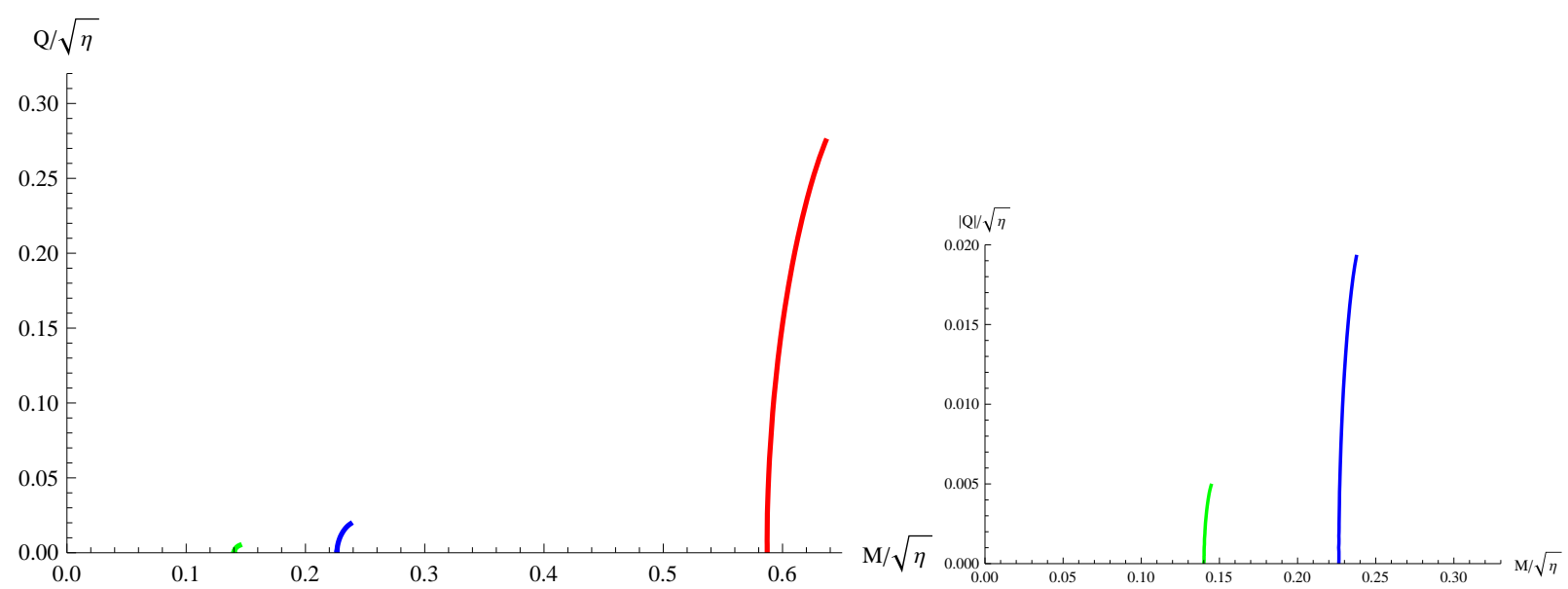

FIG. 4. The absolute value of the scalar charge $|Q|$ divided by $\sqrt{\eta}$ is shown as the function of the mass $M$ divided by $\sqrt{\eta}$. In the left larger panel, the red, blue, and green curves correspond to the scalarized BH solutions with zero, one, and two nodes of the scalar field, respectively. The right smaller panel corresponds to the enlarged display for the scalarized BH solutions with one and two nodes. We note that for $\psi_{0}>0, Q>0$ for the scalarized BH solutions with an even number of nodes, and $Q<0$ for those with an odd number of nodes. For all the cases, as the value of $\psi_{0}$ increases, the value of $|Q|$ also increases.

In Ref. [53], it was argued that all the Schwarzschild and scalarized BH solutions in the scalar-tensor theories with the quadratic coupling (10) are unstable against the radial perturbation $(\ell=0)$. It was also shown that a scalarized BH solution with $n(\geq 0)$ nodes possesses $(n+1)$ pure imaginary modes. As we argued in Sec. IC 2 , the existence of more pure imaginary modes for the scalarized BHs with more nodes can be understood in terms of the behavior of the effective potential in the test field analysis (15). For the scalarized BH solution with more nodes of the scalar field, the effective potential for the radial perturbation, Eq. (15) with $\ell=0$, contains a deeper negative region in the vicinity of the horizon for a larger positive value of $\eta / M^{2}$. In Sec. IC 3, we speculated about the way to construct stable scalarized BH solutions by adding higher order powers of $\phi$ to the GB coupling function. In Sec. III B, we will consider the general coupling function (24). We will argue the issue of the radial perturbation and stability in Sec. IV.

\section{B. General coupling}

We then consider the coupling (24). In the limit of $\alpha=0$, we recover the scalarized BH solutions obtained in Sec. III A. On the other hand, in the limit of $\alpha \gg 1$ we obtain the pure quartic order coupling (29). We will focus on the case of $\eta>0$, but not specify the sign of $\alpha$. From Eq. (51), we obtain the bound

$$
\alpha \psi_{0}^{2}>-\frac{1}{2}
$$

which is the same as Eq. (28) in the test field analysis in Sec. IC 3. We note that for the coupling (24), in addition to the Schwarzschild solution with $\psi=0$, for $\alpha<0$ those with $\psi= \pm \sqrt{-1 /(2 \alpha)}$ are also the solutions of the theory (2), as Eq. (26) in the test field analysis.

In Fig. 6 , for the scalarized BHs with zero nodes of the scalar field, the amplitude $\psi(r)$, the effective energy density $\rho^{(\mathrm{eff})}(r)$ multiplied by $r_{h}^{2}$, the effective radial pressure $p_{r}^{(\mathrm{eff})}(r)$ multiplied by $r_{h}^{2}$, and the effective tangential pressure $p_{t}^{\text {(eff) }}(r)$ multiplied by $r_{h}^{2}$ are shown as the functions of $r / r_{h}$ for $\psi_{0}=0.01$. In each panel, the red, blue, and green curves correspond to the scalarized BH solutions for $\left(\eta / r_{h}^{2}, \alpha\right)=(0.725,0),(0.338,10000),(7.31,-4990)$, respectively. In the case of $\alpha<0, \psi(r)$ approaches zero more slowly than in the case of $\alpha=0$, while in the case of $\alpha>0$ faster than in the case of $\alpha=0$. In the vicinity of the horizon, $1.1 r_{h} \lesssim r \lesssim 1.7 r_{h}, \rho^{(\mathrm{eff})}(r)$ becomes negative for $\alpha<0$, while it becomes positive for $\alpha>0$. $p_{r}^{(\text {eff })}(r)$ is positive both for $\alpha<0$ and for $\alpha>0$, which is consistent with the analysis in Ref. [50]. In the vicinity of the horizon, $1.1 r_{h} \lesssim r \lesssim 1.8 r_{h}, p_{t}^{\text {(eff) }}(r)$ becomes negative for $\alpha<0$. In Figs. 7 and 8 , the same plots for the scalarized BH solutions with one and two nodes of the scalar field are shown, respectively. Other than the oscillatory behaviors, the essential behaviors of the scalarized BH solutions with one and two nodes of the scalar field are the same as those with zero nodes of the scalar field. 


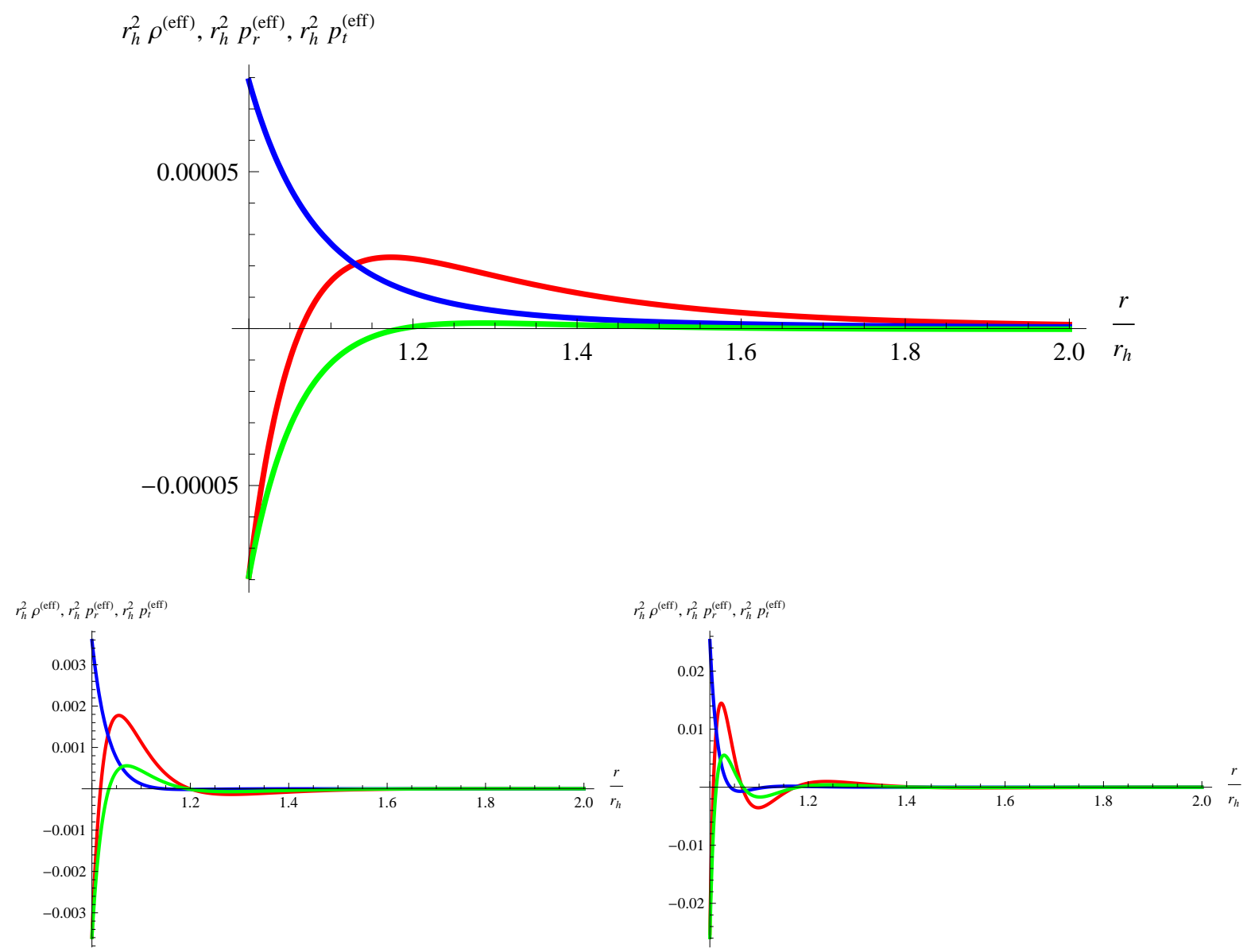

FIG. 5. The effective energy density $\rho^{(\mathrm{eff})}(r)$, the effective radial pressure $p_{r}^{(\mathrm{eff})}(r)$, and the effective tangential pressure $p_{t}^{(\mathrm{eff})}(r)$ defined in Eq. (43), which are multiplied by $r_{h}^{2}$, are shown as the functions of $r / r_{h}$ for $\psi_{0}=0.01$. The top panel corresponds to the cases of the scalarized BH solutions with zero nodes of the scalar field, while the bottom-left and bottom-right panels correspond to the cases of the scalarized $\mathrm{BH}$ solutions with one and two nodes of the scalar field, respectively. In each panel, the red, blue, and green curves correspond to $\rho^{(\mathrm{eff})}(r), p_{r}^{(\mathrm{eff})}(r)$, and $p_{t}^{(\mathrm{eff})}(r)$, respectively.

In Fig. 9, for $\alpha \geq 0$ the absolute value of the scalar charge $|Q|$ divided by $r_{h}$ is shown as the function of $\left(M / r_{h}-1 / 2\right)$, where the Schwarzschild solution without the scalar charge corresponds to the origin $(0,0)$. In the top panel, the red, blue, and green curves correspond to the scalarized $\mathrm{BH}$ solutions with zero, one, and two nodes of the scalar field, respectively. The bottom-left and bottom-right panels show the enlarged displays for the scalarized BH solutions with one and two nodes of the scalar field, respectively. In each panel, each curve represents the scalarized BH solutions for the same value of $\psi_{0}$ and the curves from the bottom correspond to those with the smaller values of $\psi_{0}$. The scalarized $\mathrm{BH}$ solutions in the pure quadratic coupling model $(\alpha=0)$ correspond to the upper edges of the red, blue, and green regions, respectively. The bottom black solid curves in the red, blue, and green regions correspond to the scalarized $\mathrm{BH}$ solutions with the same number of nodes for the pure quartic order coupling to the GB term $(\alpha \rightarrow \infty)(29)$, and the top black dashed curves correspond to those for the pure quadratic order coupling to the GB term $(\alpha=0)(10)$. In the unit of $r_{h}$, for a fixed value of $\psi_{0}$ as $\alpha$ increases positively, $M / r_{h}$ increases while $|Q| / r_{h}$ decreases.

In Fig. 9, we find that the black curve for each value for the pure quartic order coupling (29) covers only a part of the lower edge of the corresponding region for the coupling (24). This indicates that for a larger fixed value of $\psi_{0}$ the scalarized $\mathrm{BH}$ solutions exist only up to a finite value of $\alpha>0$. For a value of $\psi_{0}$ below some critical value the scalarized BH solutions exist for an arbitrarily large value of $\alpha$, and hence the limit to the pure quartic order coupling model (29) exists. The existence of such a critical value of $\psi_{0}$ can be understood in terms of the condition for the existence of the solution (45). For the coupling function (24), the condition Eq. (45) reduces to

$$
r_{h}^{4}>6 \eta^{2} \psi_{0}^{2}\left(1+2 \alpha \psi_{0}^{2}\right)^{2} .
$$

From Eq. (53) with $\psi_{0}>0$, in the regime where the quartic order term dominates the quadratic one the bound (54) 


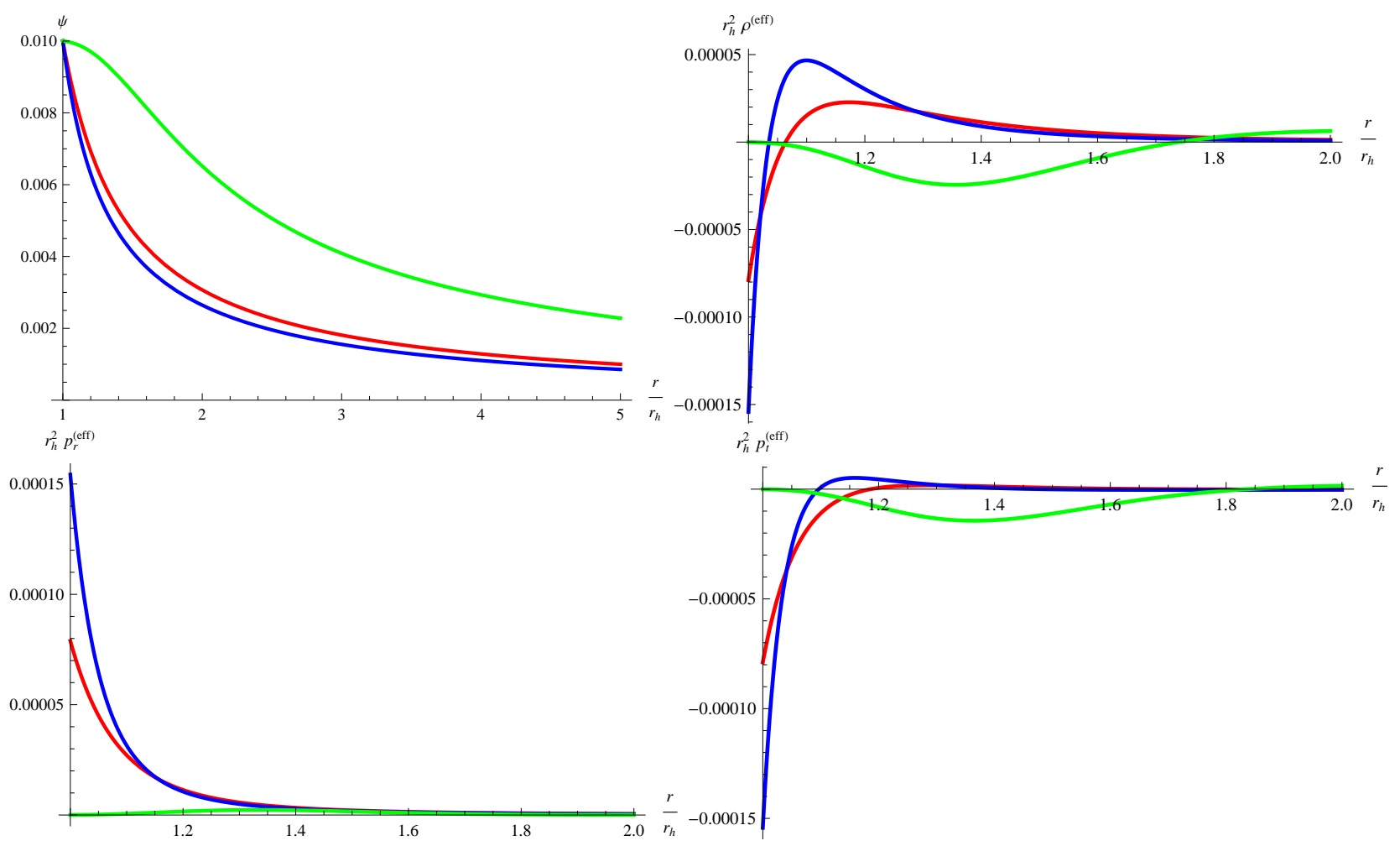

FIG. 6. For the scalarized BHs with zero nodes of the scalar field, the amplitude $\psi(r)$, the effective energy density $\rho^{(\text {eff })}(r)$ multiplied by $r_{h}^{2}$, the effective radial pressure $p_{r}^{\text {(eff) }}(r)$ multiplied by $r_{h}^{2}$, and the effective tangential pressure $p_{t}^{\text {(eff) }}(r)$ multiplied by $r_{h}^{2}$ are shown as the functions of $r / r_{h}$ for $\psi_{0}=0.01$. In each panel, the red, blue, and green curves correspond to the scalarized BH solutions for $\left(\eta / r_{h}^{2}, \alpha\right)=(0.725,0),(0.338,10000),(7.31,-4990)$, respectively.

reduces to

$$
\eta<\frac{r_{h}^{2}}{2 \sqrt{6} \alpha \psi_{0}^{3}}
$$

Assuming that the limit to the case of the pure quartic order coupling with the coupling constant $\lambda_{0}$ exists in the large $\alpha$ limit, $\eta$ and $\alpha$ giving a scalarized BH solution are related by a scaling relation $\eta=\lambda_{0} / \alpha$. Hence, the existence of the limit to the pure quartic order coupling requires $r_{h}^{2} /\left(2 \sqrt{6} \alpha \psi_{0}^{3}\right)>\lambda_{0} / \alpha$, which gives rise to the critical value of $\psi_{0}$ :

$$
\psi_{0}<\left(\frac{r_{h}^{2}}{2 \sqrt{6} \lambda_{0}}\right)^{\frac{1}{3}}
$$

In Fig. 10, the scalar charge $Q$ divided by $\sqrt{\eta}$ for scalarized $\mathrm{BH}$ solutions with zero nodes of the scalar field is shown as the function of the mass $M$ divided by $\sqrt{\eta}$ for fixed values of $\alpha$. The red, blue, green, black, cyan, magenta, and brown curves correspond to the cases of $\alpha=20000,2000,100,0,-10,-100,-1000$, respectively. The dashed curves represent scalarized $\mathrm{BH}$ solutions for each constant value of $\psi_{0}$ for $\alpha>0$, where the upper curves correspond to the larger values of $\psi_{0}$. For all values of $\alpha$, the branches of the scalarized BH solutions with zero nodes bifurcate from the Schwarzschild solution $Q=0$, at $M / \sqrt{\eta}=0.587$ for $\psi_{0}=0$, and as $\psi_{0}$ increases, the scalar charge $|Q| / \sqrt{\eta}$ increases. This confirms our argument in Sec. IC 1 that the onset of the tachyonic instability of the Schwarzschild solution is determined by the quadratic order term in $\xi(\phi)$. For $\alpha<0$, we have chosen the maximal value of $\psi_{0}$ to $\sqrt{-1 /(2 \alpha)}\left(1-10^{-8}\right)$.

In Fig. 11, the absolute value of the scalar charge $|Q|$ divided by $\sqrt{\eta}$ is shown as the function of the mass $M$ divided by $\sqrt{\eta}$ for $\psi_{0}=0.005$, for which all the scalarized $\mathrm{BH}$ solutions with zero, one, and two nodes of the scalar field exist. The red, blue, and green curves correspond to the scalarized BH solutions with zero, one, and two nodes of the scalar field, respectively. In the limit of $\alpha \rightarrow \infty$, the red and blue curves for the scalarized BH solutions with zero and one 


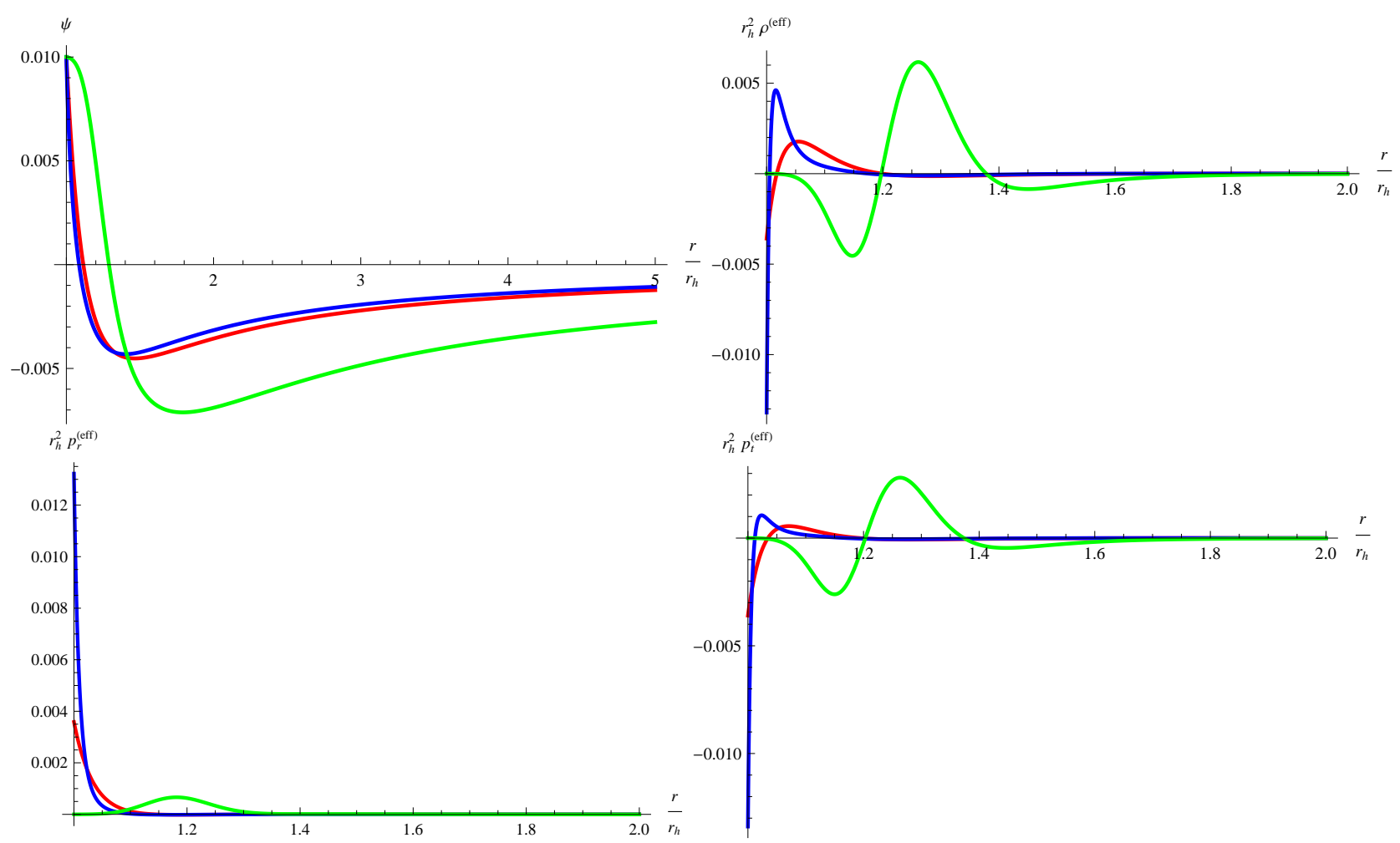

FIG. 7. For the scalarized BHs with one node of the scalar field, the amplitude $\psi(r)$, the effective energy density $\rho^{(\text {eff })}(r)$ multiplied by $r_{h}^{2}$, the effective radial pressure $p_{r}^{\text {(eff) }}(r)$ multiplied by $r_{h}^{2}$, and the effective tangential pressure $p_{t}^{\text {(eff) }}(r)$ multiplied by $r_{h}^{2}$, are shown as the functions of $r / r_{h}$ for $\psi_{0}=0.01$. In each panel, the red, blue, and green curves correspond to the scalarized $\mathrm{BH}$ solutions for $\left(\eta / r_{h}^{2}, \alpha\right)=(4.87,0),(3.09,10000),(20.2,-4990)$, respectively.

node approach the straight lines corresponding to the scalarized BH solutions with the same number of nodes for the pure quartic coupling (29). We find that the relation between $M$ and $Q$ is very similar to Fig. 2 in Sec. IC for the test field analysis, except for the existence of the upper bound on $\alpha$ for the scalarized BH solutions with two nodes. We expect that the reason why the full analysis is similar to the test field analysis is because of the existence of the condition (54), which does not allow a large value of $\psi_{0}$ for which the backreaction would spoil the essential features obtained from the analysis in Sec. IC.

In Fig. 12, for scalarized BHs with zero nodes of the scalar field, $\eta / M^{2}$ is shown as the function of $\alpha$ for several values of $\psi_{0}=\mathcal{O}(0.01)$. The red, blue, and green curves correspond to the cases of $\psi_{0}=0.005,0.01$, and 0.03 , respectively. For a given $\psi_{0}$, as $\alpha$ increases, $\eta / M^{2}$ decreases. The value of $\eta / M^{2}$ blows up at $\alpha=-1 /\left(2 \psi_{0}^{2}\right)$, where the bound (53) is saturated. We note that for a solar-mass $\mathrm{BH}$ with $M=\mathcal{O}\left(G M_{\odot} / c^{2}\right)=\mathcal{O}(1 \mathrm{~km}), \eta / M^{2}>1$ if $\sqrt{\eta^{-1}}<10^{-11} \mathrm{eV}$, and for a BH with $M=\mathcal{O}\left(\left(G / c^{2}\right) 10^{15} \mathrm{~g}\right)=\mathcal{O}\left(10^{-13} \mathrm{~cm}\right)$, which is considered as the lowest mass of primordial BHs [55], $\eta / M^{2}>1$ if $\sqrt{\eta^{-1}}<10^{8} \mathrm{eV}$, for which the scalarized BHs can be realized also for $\alpha<0$.

In Sec. IV, we will analyze the radial perturbation of the scalarized BH solutions obtained in this section. We will find that the similarity of the full analysis to the test field one will also hold at the level of the linear perturbation, and scalarized BHs which are radially stable exist only for the case of $\alpha<0$.

\section{RADIAL PERTURBATION}

In this section, we will analyze the radial perturbation about the scalarized BHs obtained in Sec. III. 


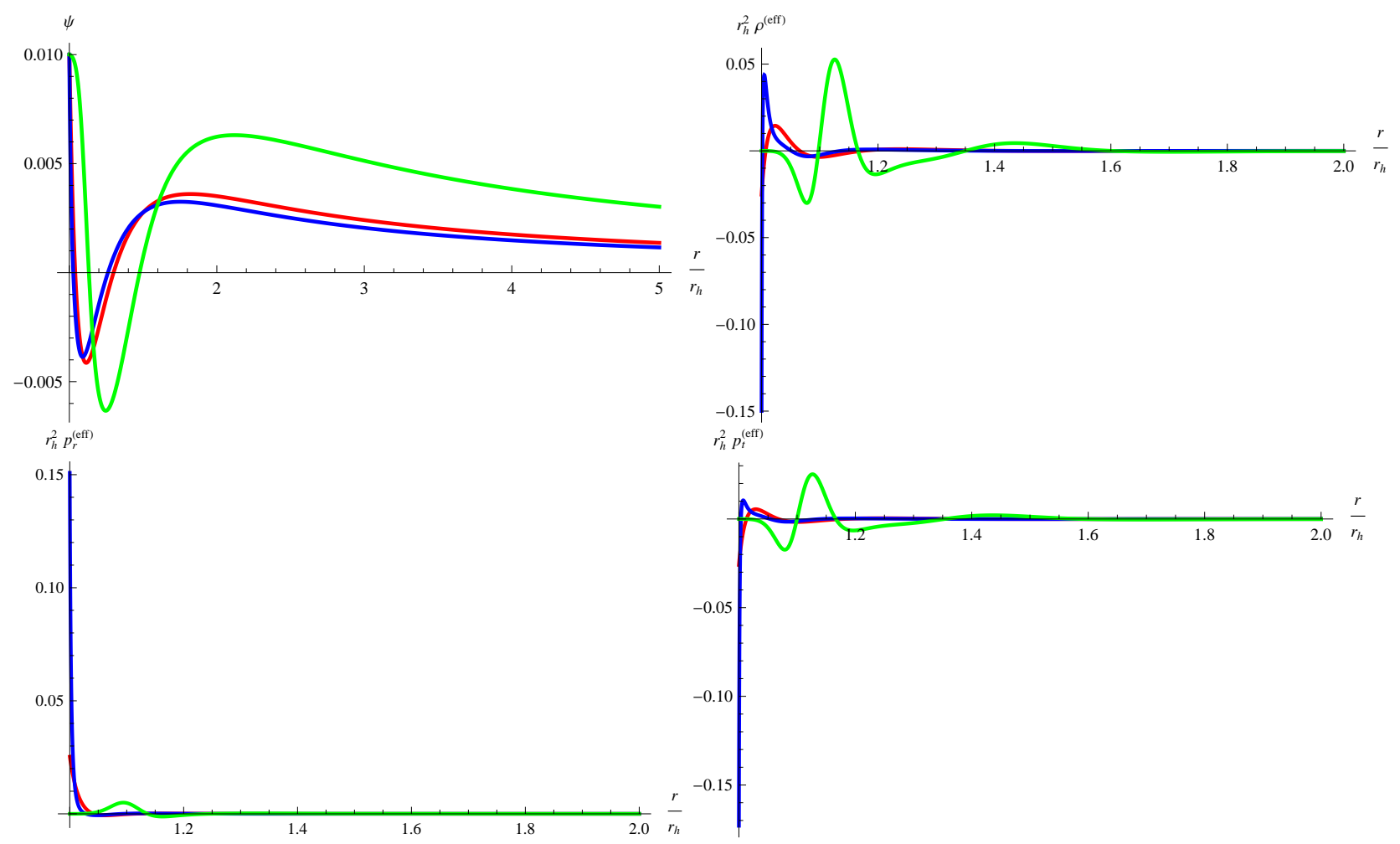

FIG. 8. For the scalarized BHs with two nodes of the scalar field, the amplitude $\psi(r)$, the effective energy density $\rho^{(\text {eff })}(r)$ multiplied by $r_{h}^{2}$, the effective radial pressure $p_{r}^{\text {(eff) }}(r)$ multiplied by $r_{h}^{2}$, and the effective tangential pressure $p_{t}^{\text {(eff) }}(r)$ multiplied by $r_{h}^{2}$ are shown as the functions of $r / r_{h}$ for $\psi_{0}=0.01$. In each panel, the red, blue, and green curves correspond to the scalarized $\mathrm{BH}$ solutions for $\left(\eta / r_{h}^{2}, \alpha\right)=(12.7,0),(9.19,10000),(36.2,-4990)$, respectively.

\section{A. Master equation}

We start from the general time-dependent spherically symmetric ansatz of the spacetime and the scalar field:

$$
\begin{aligned}
d s^{2} & =-\tilde{A}(t, r) d t^{2}+\frac{d r^{2}}{\tilde{B}(t, r)}+r^{2}\left(d \theta^{2}+\sin ^{2} \theta d \varphi^{2}\right), \\
\phi & =\phi(t, r) .
\end{aligned}
$$

We assume that the deviation from a static and spherically symmetric BH solution is small,

$$
\tilde{A}=A(r)+\varepsilon a(t, r), \quad \tilde{B}=B(r)+\varepsilon b(t, r), \quad \phi=\psi(r)+\varepsilon \Phi(t, r),
$$

where $(A, B, \psi)$ represents a background $\mathrm{BH}$ solution and $(a, b, \Phi)$ does the perturbation about it. Expanding up to the first order of $\varepsilon(\ll 1)$, the perturbed $(t, t)-,(r, r)-,(t, r)-$, and angular components of the gravitational equation of motion Eq. (3) and the perturbed scalar field equation of motion Eq. (7) are, respectively, obtained as

$$
\begin{aligned}
& \alpha_{1} \Phi^{\prime \prime}+\alpha_{2} \Phi^{\prime}+\alpha_{3} \Phi+\alpha_{4} b+\alpha_{5} b^{\prime}=0, \\
& \beta_{1} \ddot{\Phi}+\beta_{2} \Phi^{\prime}+\beta_{3} \Phi+\beta_{4} a+\beta_{5} a^{\prime}+\beta_{6} b=0, \\
& \gamma_{1} \dot{b}+\gamma_{2} \dot{\Phi}^{\prime}+\gamma_{3} \dot{\Phi}=0, \\
& c_{1} \ddot{\Phi}+c_{2} \Phi^{\prime \prime}+c_{3} \Phi^{\prime}+c_{4} \Phi+c_{5} a^{\prime \prime}+c_{6} a^{\prime}+c_{7} a+c_{8} \ddot{b}+c_{9} b^{\prime}+c_{10} b=0, \\
& d_{1} \ddot{\Phi}+d_{2} \Phi^{\prime \prime}+d_{3} \Phi^{\prime}+d_{4} \Phi+d_{5} a^{\prime \prime}+d_{6} a^{\prime}+d_{7} a+d_{8} \ddot{b}+d_{9} b^{\prime}+d_{10} b=0,
\end{aligned}
$$

where a "dot" denotes the derivative with respect to the time $t$, and $\alpha_{i}(i=1,2, \cdots, 5), \beta_{i}(i=1,2, \cdots, 6), \gamma_{i}$ $(i=1,2,3), c_{i}(i=1,2, \cdots, 10)$, and $d_{i}(i=1,2, \cdots, 10)$ are the functions of $r$, which are given in Appendix A. 


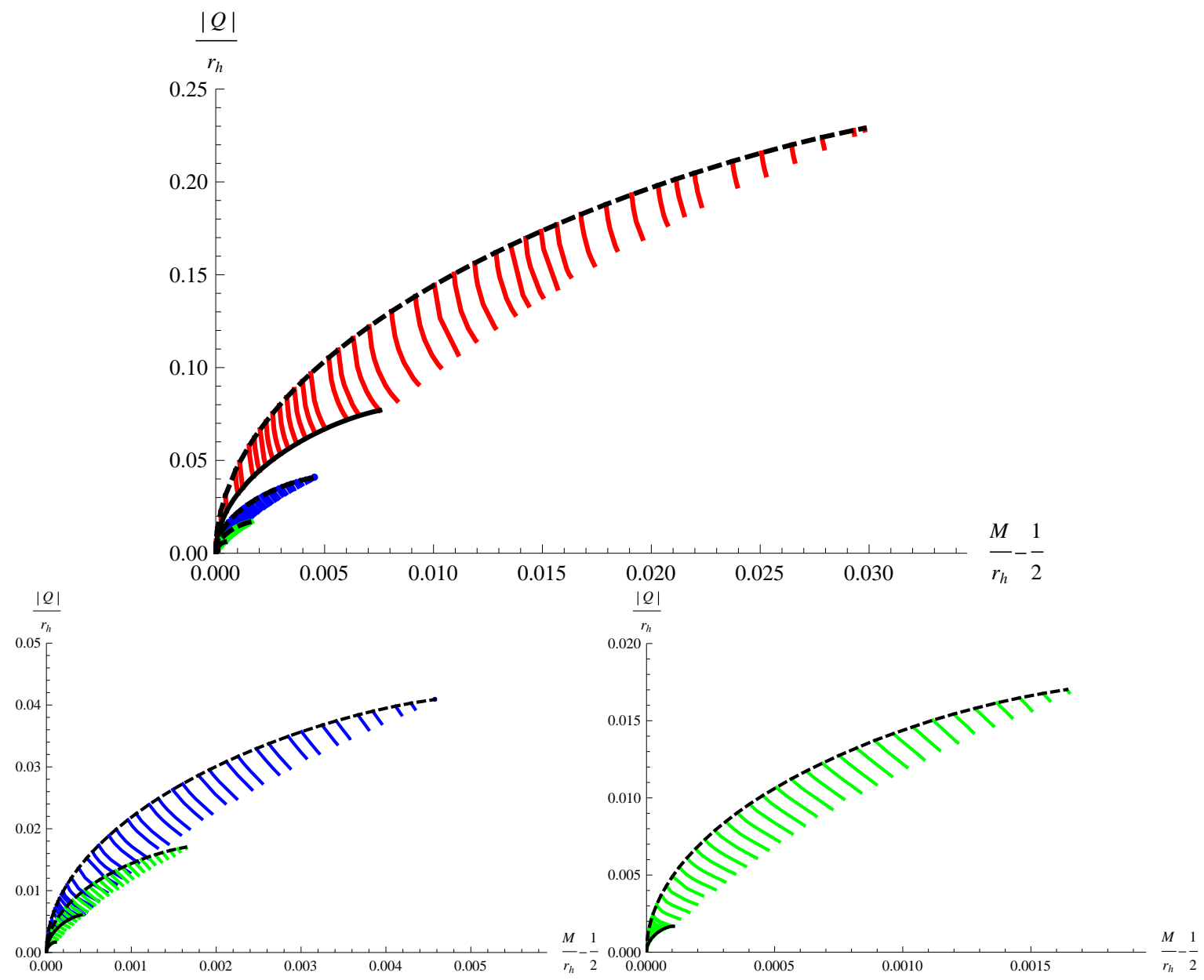

FIG. 9. The absolute value of the scalar charge $|Q|$ divided by $r_{h}$ is shown as the function of $\left(M / r_{h}-1 / 2\right)$. The Schwarzschild solution without the scalar charge corresponds to the origin $(0,0)$. In the top panel, the red, blue, and green curves correspond to the scalarized BH solutions with zero, one, and two nodes of the scalar field, respectively. The bottom-left and bottom-right small panels show the enlarged displays for the scalarized BH solutions with one and two nodes of the scalar field, respectively. In each panel, each curve represents the scalarized $\mathrm{BH}$ solutions for the same value of $\psi_{0}$ and the curves from the bottom correspond to those with the smaller values of $\psi_{0}$. The scalarized $\mathrm{BH}$ solutions in the pure quadratic coupling model $(\alpha=0)$ correspond to the upper edges of the red, blue, and green regions, respectively. The bottom black solid curves in the red, blue, and green regions correspond to the scalarized BH solutions with the same number of nodes for the pure quartic order coupling to the GB term $(\alpha \rightarrow \infty)$ (29), and the top black dashed curves correspond to those for the pure quadratic order coupling to the GB term $(\alpha=0)(10)$. We note that for $\psi_{0}>0, Q>0$ for the scalarized BH solutions with an even number of nodes, and $Q<0$ for those with an odd number of nodes.

Integrating (62) with respect to $t$,

$$
b=-\frac{1}{\gamma_{1}}\left(\gamma_{2} \Phi^{\prime}+\gamma_{3} \Phi\right),
$$

where the integration constant is set to zero by a redefinition of the background solution. Plugging Eq. (65) into Eq. (61), $a^{\prime}$ can be expressed in terms of $a, \Phi, \Phi^{\prime}$, and $\ddot{\Phi}$ :

$$
a^{\prime}=-\frac{\beta_{1}}{\beta_{5}} \ddot{\Phi}+\frac{-\beta_{2} \gamma_{1}+\beta_{6} \gamma_{2}}{\beta_{5} \gamma_{1}} \Phi^{\prime}+\frac{-\beta_{3} \gamma_{1}+\beta_{6} \gamma_{3}}{\beta_{5} \gamma_{1}} \Phi-\frac{\beta_{4}}{\beta_{5}} a .
$$

Multiplying Eqs. (63) and (64) by $d_{5}$ and $c_{5}$, respectively, and taking their difference, the term proportional to $a^{\prime \prime}$ is eliminated. Substituting Eqs. (65) and (66) into it and eliminating $a^{\prime}$ and $b$ (and its derivatives), we finally arrive in the master equation for the radial perturbation:

$$
-\rho_{1} \ddot{\Phi}+\rho_{2} \Phi^{\prime \prime}+\rho_{3} \Phi^{\prime}+\rho_{4} \Phi=0,
$$




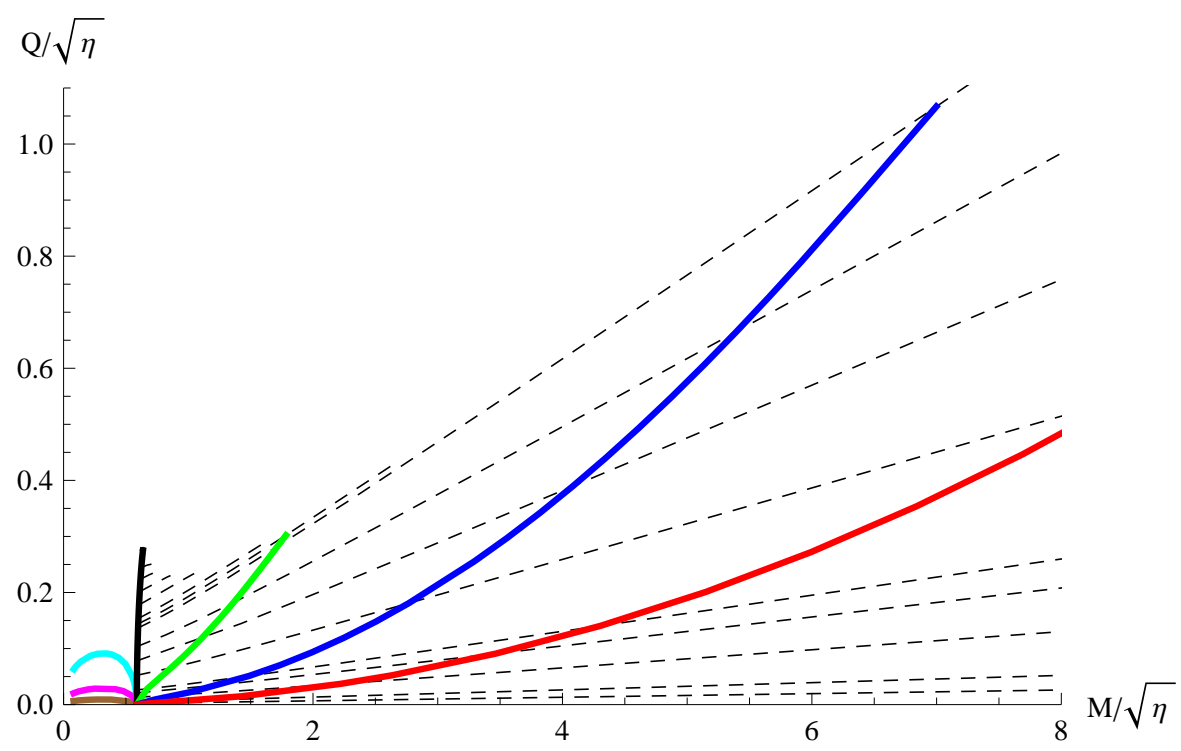

FIG. 10. The scalar charge $Q$ divided by $\sqrt{\eta}$ for scalarized BH solutions with zero nodes of the scalar field is shown as the function of the mass $M$ divided by $\sqrt{\eta}$ for fixed values of $\alpha$. The red, blue, green, black, cyan, magenta, and brown curves correspond to the cases of $\alpha=20000,2000,100,0,-10,-100,-1000$, respectively. The dashed curves represent scalarized BH solutions for each constant value of $\psi_{0}$ for $\alpha>0$, where the upper curves correspond to the larger values of $\psi_{0}$. For $\alpha<0$, we have chosen the maximal value of $\psi_{0}$ to $\sqrt{-1 /(2 \alpha)}\left(1-10^{-8}\right)$.

where $\rho_{i}(i=1,2,3,4)$ are given in Appendix A. Assuming that $\Phi=e^{-i \omega t} \Phi_{\omega}(r)$ with $\Phi_{\omega}=C(r) \Psi_{\omega}(r)$ where the function $C(r)$ satisfies

$$
\frac{C^{\prime}}{C}=\frac{1}{4}(\ln (A B))^{\prime}-\frac{\rho_{3}}{2 \rho_{2}}
$$

each mode satisfies the eigen equation

$$
\left[-\frac{d^{2}}{d r_{*}^{2}}+U_{\mathrm{eff}}(r)\right] \Psi_{\omega}(r)=\frac{\rho_{1}}{\rho_{2}}(A B) \omega^{2} \Psi_{\omega}(r)
$$

where we have introduced the tortoise radial coordinate $d r_{*}:=d r / \sqrt{A B}$, and the effective potential

$$
U_{\text {eff }}(r):=-A B\left\{\frac{1}{4}(\ln (A B))^{\prime \prime}+\frac{1}{16}\left[(\ln (A B))^{\prime}\right]^{2}-\frac{1}{2}\left(\frac{\rho_{3}}{\rho_{2}}\right)^{\prime}-\frac{\rho_{3}^{2}}{4 \rho_{2}^{2}}+\frac{\rho_{4}}{\rho_{2}}\right\} .
$$

A scalarized BH solution is unstable, if Eq. (69) with Eq. (70) admit pure imaginary modes $\omega^{2}<0$. On the other hand, the absence of pure imaginary modes can be ensured, if the effective potential $U_{\text {eff }}(r)$ is non-negative everywhere outside the horizon $r \geq r_{h}, U_{\text {eff }}(r) \geq 0$. In Ref. [53], the radial perturbation of the scalarized BHs in the scalar-tensor theory with the quadratic order coupling to the GB term (10) was analyzed, and the existence of pure imaginary modes was clarified, indicating that the scalarized BH solutions for these coupling functions are unstable. In the next subsection, we will consider the case of the more general coupling (24).

\section{B. Effective potential and stability}

We then apply the formulation in Sec. IV A for analyzing the stability of the scalarized BH solutions obtained in Sec. III against the radial perturbation.

In the top panel of Fig. 13, the effective potential for the radial perturbation $U_{\text {eff }}(r)$, Eq. (70), multiplied by $r_{h}^{2}$, is shown as the function of $r / r_{h}$ for the scalarized BH solutions with zero nodes of the scalar field for $\psi_{0}=0.005$. The black, red, blue, magenta, and green curves correspond to the cases of the scalarized BH solutions for $\left(\eta / r_{h}^{2}, \alpha\right)=$ 


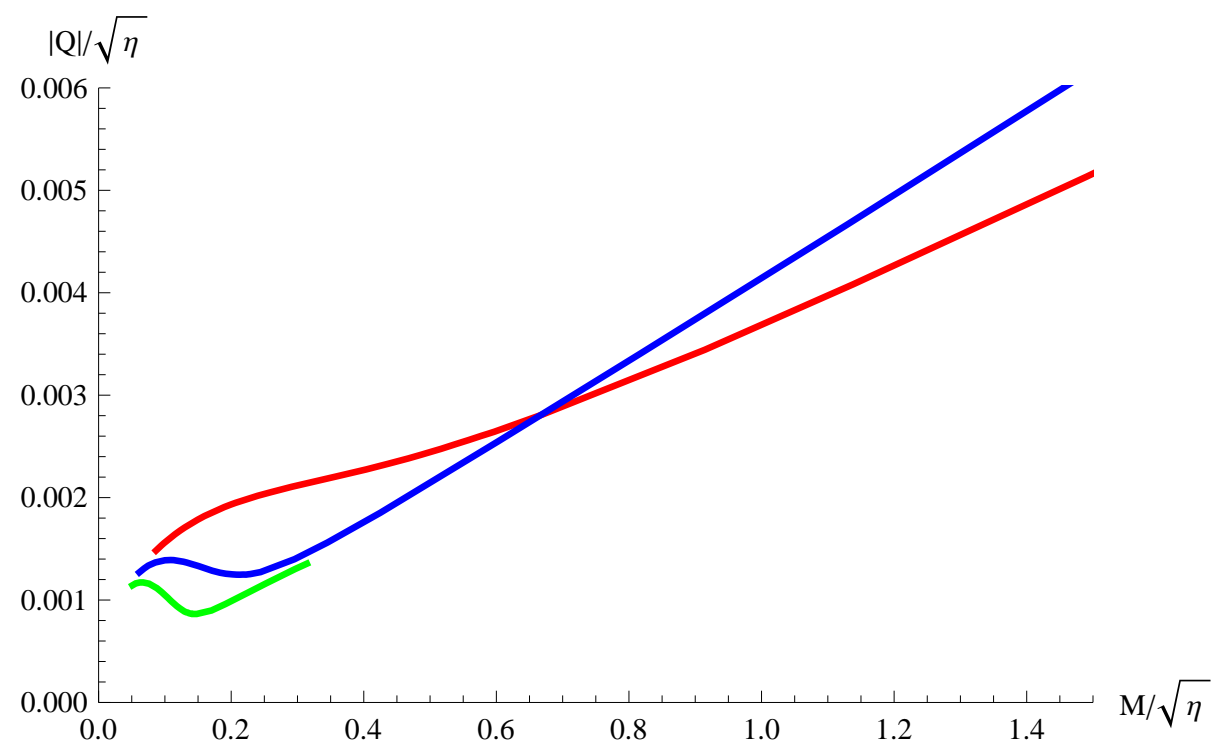

FIG. 11. The absolute value of the scalar charge $|Q|$ divided by $\sqrt{\eta}$ is shown as the function of the mass $M$ divided by $\sqrt{\eta}$ for $\psi_{0}=0.005$. The red, blue and green curves correspond to the solutions of the scalar field with zero, one, and two nodes, respectively. In the limit of $\alpha \rightarrow \infty$, the red and blue curves for the scalarized BH solutions with zero and one node approach the straight lines corresponding to the scalarized $\mathrm{BH}$ solutions with the same number of nodes for the pure quartic coupling (29). For $\alpha<0$, we have chosen the minimal value of $\alpha$ to $\left(1 / \psi_{0}^{2}\right)\left(-0.5+10^{-7}\right)$.

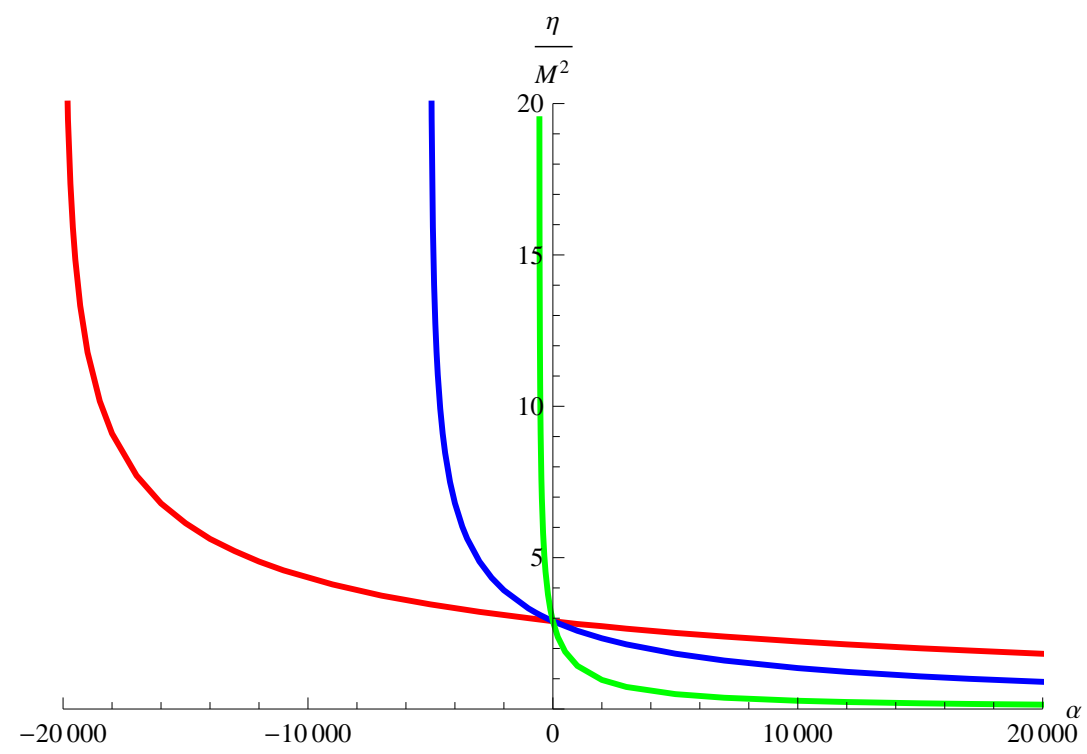

FIG. 12. For scalarized BHs with zero nodes of the scalar field, $\eta / M^{2}$ is shown as the function of $\alpha$ for several values of $\psi_{0}=\mathcal{O}(0.01)$. The red, blue, and green curves correspond to the cases of $\psi_{0}=0.005,0.01$, and 0.03 , respectively.

$(0.558,10000),(0.725,0),(0.803,-3000),(0.8505,-4619),(1.03,-9000)$, respectively. In the case of $\alpha=0, U_{\text {eff }}(r)$ contains a negative region in the vicinity of the horizon $r=r_{h}$, and the analysis in Ref. [53] suggests that it gives rise to a pure imaginary mode. In the case of $\alpha>0$, as shown in the black curve in the top panel of Fig. 13, the depth of the negative region of $U_{\text {eff }}(r)$ becomes larger than in the case of $\alpha=0$, and we speculate that the scalarized $\mathrm{BH}$ solutions obtained for $\alpha>0$ are also unstable against the radial perturbation of those in the case of $\alpha=0$.

On the other hand, in the case of $\alpha<0$, the minimum of $U_{\text {eff }}(r)$ in the vicinity of the horizon $r=r_{h}$ increases and for a sufficiently large negative value of $\alpha$ no negative region appears, as in the case of the green curve in the top panel of Fig. 13. Thus, only for a large negative quartic order coupling constant, the scalarized BHs with zero nodes 


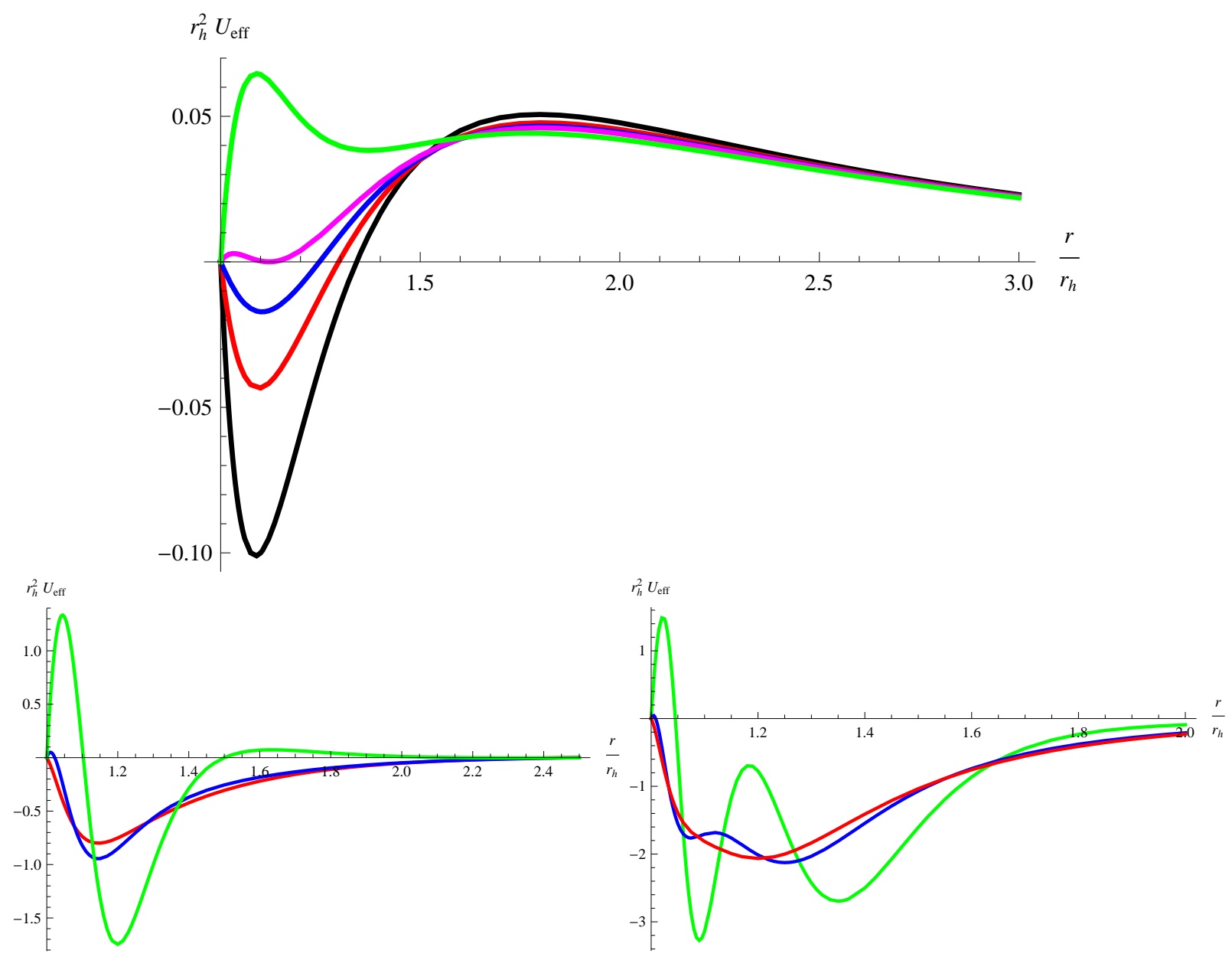

FIG. 13. In the top panel, the effective potential $U_{\text {eff }}(r)$ multiplied by $r_{h}^{2}$ is shown as the function of $r / r_{h}$ for the scalarized $\mathrm{BH}$ solutions with zero nodes of the scalar field for $\psi_{0}=0.005$. The black, red, blue, magenta, and green curves correspond to the cases of the scalarized BHs for $\left(\eta / r_{h}^{2}, \alpha\right)=(0.558,10000),(0.725,0),(0.803,-3000),(0.8505,-4619),(1.03,-9000)$, respectively. Similarly, the bottom-left and bottom-right panels describe the effective potential $U_{\text {eff }}(r)$ multiplied by $r_{h}^{2}$ as the function of the mass $r / r_{h}$ for the scalarized BH solutions with one and two nodes of the scalar field for $\psi_{0}=0.005$, respectively. In the bottom-left and bottom-right panels, the red, blue, and green curves correspond to the cases of for the scalarized $\mathrm{BH}$ solutions with one node for $\left(\eta / r_{h}^{2}, \alpha\right)=(5.27,-4000),(6.12,-10000),(11.0,-19000)$, and for the scalarized BH solutions with two nodes for $\left(\eta / r_{h}^{2}, \alpha\right)=(13.4,-4000),(14.9,-10000),(22.9,-19000)$, respectively.

of the scalar field are expected to become stable against the radial perturbation. As shown by the magenta curve in the top panel of Fig. 13, the minimum of $U_{\text {eff }}(r)$ becomes zero for $\alpha \approx-4619$ in the case of $\psi_{0}=0005$. Hence, there is no negative region in $U_{\text {eff }}(r)$ and the scalarized $\mathrm{BH}$ solutions with zero nodes of the scalar field are expected to be stable for $-20000<\alpha \lesssim-4619$. Moreover, for $\psi_{0}=0.005$ and $\alpha \approx-4619$, we find that $\alpha \psi_{0}^{2} \approx 0.1155$, which almost agreed with the upper bound of Eq. (31) in our test field analysis in Sec. IC. Thus, at least for a small value of $\psi_{0}$, the scalarized BH solutions with zero nodes of the scalar field which are stable against the radial perturbation exist almost for the range given by Eq. (31).

In the bottom-left and bottom-right panels of Fig. 13, the red, blue, and green curves correspond to $U_{\text {eff }}(r)$ for $\left(\eta / r_{h}^{2}, \alpha\right)=(5.27,-4000),(6.12,-10000),(11.0,-19000)$ for the scalarized BH solutions with one node, and for $\left(\eta / r_{h}^{2}, \alpha\right)=(13.4,-4000),(14.9,-10000),(22.9,-19000)$ for the scalarized BH solutions with two nodes, respectively. We find that in the cases of the scalarized BHs with one and two nodes of the scalar field, even for a negative value of $\alpha$, the effective potential $U_{\text {eff }}(r)$ always contains a negative region. Thus, we conclude that in the case of the coupling to the GB term (24) the scalarized BH solutions with one and two nodes are always unstable against the radial perturbation. We note that for all the cases shown in Fig. 13, $\rho_{1}>0$ and $\rho_{2}>0$ in Eq. (67), and, hence, there is no violation of the hyperbolicity of the radial perturbation, at least for the given choice of the scalarized $\mathrm{BH}$ solutions. 
The above features are quite similar to our perspectives obtained from the test field analysis in Sec. IC. As we argued in Sec. III B, we speculate that this similarity is due to the presence of the bound (54), namely, the amplitude of the scalar field cannot be so large that the backreaction on the metric significantly modifies features of the scalarized BHs. As expected, for the Schwarzschild solution with the constant scalar field $\psi(r)= \pm \sqrt{-1 /(2 \alpha)}$ for $\alpha<0$, the effective potential $U_{\text {eff }}(r)$ agrees with Eq. (32) obtained in the test field analysis, which contains no negative region.

Thus, we conclude that among the scalarized BH solutions in the scalar-tensor theory (24) only the scalarized BH solutions with zero nodes for a sufficiently large negative value of $\alpha<0$ can be stable against the radial perturbation. We have to emphasize, however, that the radial stability does not ensure the stability of BHs against the other perturbations. They should be analyzed separately in the future work.

\section{CONCLUSIONS}

We have investigated the scalarized black hole $(\mathrm{BH})$ solutions in the scalar-tensor theory with the coupling of the scalar field to the Gauss-Bonnet (GB) term (2). In Sec. I, after reviewing how the spontaneous scalarization may take place around a vacuum BH due to a tachyonic instability triggered by the coupling to the GB term which is analogous to that inside relativistic stars triggered by the coupling to the matter field, we have demonstrated such a possibility by a test field analysis. We have obtained the nontrivial solution of a static test scalar field which is coupled to the curvature of the background Schwarzschild spacetime. We have shown that around a Schwarzschild BH there are the nontrivial static scalar field solutions with zero, one, and two nodes (and there should be those with more nodes which were not investigated in this paper) for the pure quadratic order coupling (10) and the general coupling (24). We have also analyzed the radial scalar field perturbation of these nontrivial scalar field solutions, and confirmed the existence of a negative region of the effective potential of the scalar field perturbation. Only the exceptional case was the scalar field solution with zero nodes for a sufficiently large negative value of the quartic order coupling satisfying Eq. (31), where there is no negative region in the effective potential and hence no pure imaginary mode.

In Sec. II, we have explained our strategy for constructing the static and spherically symmetric scalarized BH solutions in the scalar-tensor theory with an arbitrary coupling to the GB term. By arranging the components of the gravitational equations of motion (35) and (36) and the scalar field equation of motion (37), we have obtained a set of the equations (40) and (41) to determine the time component of the metric $A(r)$ and the scalar field $\psi(r)$. With use of the solution for $A(r)$ and $\psi(r)$, the radial component of the metric $B(r)$ was then determined via Eq. (38). We have focused on the scalarized BH solutions whose asymptotic value of the scalar field at the infinity is zero, which may be the endpoint of the tachyonic instability of the Schwarzschild BH with the constant scalar field $\psi(r)=0$.

In Sec. III, we have numerically constructed the scalarized BH solutions in the scalar-tensor theories with the pure quadratic order coupling (10) and the more general coupling (24) to the GB term. The case of the pure quadratic order coupling has been already analyzed in Ref. [48], and the results were recovered in our analysis. In the case of the more general coupling (24), we numerically confirmed the existence of the scalarized BH solutions with zero, one, two nodes (and there should be the solutions with more nodes), as well as the Schwarzschild BH solutions with the constant scalar field $\psi(r)=0$ and $\psi(r)= \pm \sqrt{-1 /(2 \alpha)}$, where the latter exists only for $\alpha<0$. In the limit of $\alpha \gg 1$, if the amplitude of the scalar field at the horizon $\psi_{0}$ is below the certain critical value given by Eq. (56), the scalarized $\mathrm{BH}$ solutions approach those in the case of the pure quartic order coupling (29). If $\psi_{0}$ is larger than the value (56), there is the maximal value of $\alpha$ above which there is no scalarized BH solution and hence no limit to the pure quartic order coupling model. For $\alpha<0$, there were also the scalarized BH solutions, but, in order to satisfy the boundary condition, $\alpha$ had to satisfy the bound (53) for a given $\psi_{0}$.

In Sec. IV, we have investigated the radial perturbation of the scalarized BHs obtained in Sec. III. The combination of the nontrivial components of the gravitational equations of motion and the scalar field equation of motion resulted in the single master equation (67). We have confirmed that in the case of the pure quadratic order coupling the effective potential for the radial perturbation (70) has a negative region in the vicinity of the horizon $r=r_{h}$ for all the scalarized BHs with zero, one, and two nodes, indicating the existence of pure imaginary modes. We have shown that in the case of the general coupling (24) with the negative quartic order coupling constant $\alpha<0$, only for the scalarized BH solutions with zero nodes of the scalar field the effective potential becomes non-negative in the vicinity of the horizon $r=r_{h}$, implying the absence of pure imaginary modes. This was consistent with the test field analysis performed in Sec. IC. We also expected that the region where the scalarized BH solutions are stable against the radial perturbation coincides with that obtained in the test field analysis, Eq. (31), at least for a smaller value of $\psi_{0}$. On the other hand, the scalarized BH solutions with more than one node of the scalar field were always expected to be unstable against the radial perturbation.

There will be a number of subjects related to our work. First, for the scalarized BH solutions which were expected to be stable against the radial perturbation, in order to clarify the complete stability, it will be necessary to analyze the other type of perturbations. On the other hand, in the case that the scalarized BH solutions are unstable against 
the radial perturbation, the scalarized $\mathrm{BHs}$ would not be the fate of gravitational collapse. In such a case, it will be very interesting to develop a numerical scheme to follow gravitational collapse of the scalar field. Finally, it will also be interesting to consider the scalarization of a vacuum BH solution in the presence of the coupling of the scalar field to other invariants, such as the Pontryagin density ${ }^{*} R^{\mu \nu \alpha \beta} R_{\mu \nu \alpha \beta}$ and the Maxwell term $F_{\mu \nu} F^{\mu \nu}[56]$ (See also for the earlier works on the coupling to the Born-Infeld term [57, 58]). We will come back to these issues in future publications.

\section{ACKNOWLEDGMENTS}

We thank Thomas Sotiriou for helpful discussions. M. M. was supported by FCT-Portugal through Grant No. SFRH/BPD/88299/2012. T. I. acknowledges financial support provided under the European Union's H2020 ERC Consolidator Grant "Matter and strong- field gravity: New frontiers in Einstein's theory" grant agreement no. MaGRaTh-646597, and under the H2020-MSCA-RISE-2015 Grant No. StronGrHEP-690904.

\section{Appendix A: Coefficients}

In this appendix, we show the coefficients in the equations for the perturbative analysis shown in Sec. IV.

The coefficients in Eq. (60) are given by

$$
\begin{aligned}
& \alpha_{1}=4(B-1) B \xi^{(1)}(\psi), \\
& \alpha_{2}=\frac{1}{2}\left(4(3 B-1) B^{\prime} \xi^{(1)}(\psi)+B \psi^{\prime}\left(-r^{2}+16(B-1) \xi^{(2)}(\psi)\right)\right), \\
& \alpha_{3}=2\left[(3 B-1) B^{\prime} \psi^{\prime} \xi^{(2)}(\psi)+2(B-1) B\left(\xi^{(2)}(\psi) \psi^{\prime \prime}+\psi^{\prime 2} \xi^{(3)}(\psi)\right)\right], \\
& \alpha_{4}=\frac{1}{4}\left[24 B^{\prime} \xi^{(1)}(\psi) \psi^{\prime}-\psi^{\prime 2}\left(r^{2}+16(1-2 B) \xi^{(2)}(\psi)\right)+4\left(-1+4(2 B-1) \xi^{(1)}(\psi) \psi^{\prime \prime}\right)\right], \\
& \alpha_{5}=-\left(r+2(1-3 B) \xi^{(1)}(\psi) \psi^{\prime}\right) .
\end{aligned}
$$

The coefficients in Eq. (61) are given by

$$
\begin{aligned}
& \beta_{1}=-\frac{4(B-1) \xi^{(1)}(\psi)}{A}, \\
& \beta_{2}=\frac{B}{2 A}\left[4(3 B-1) A^{\prime} \xi^{(1)}(\psi)+r^{2} A \psi^{\prime}\right], \\
& \beta_{3}=\frac{2 B(3 B-1) A^{\prime} \psi^{\prime} \xi^{(2)}(\psi)}{A} \\
& \beta_{4}=\frac{B A^{\prime}}{A^{2}}\left(r+2(1-3 B) \xi^{(1)}(\psi) \psi^{\prime}\right) \\
& \beta_{5}=-\frac{B}{A}\left(r+2(1-3 B) \xi^{(1)}(\psi) \psi^{\prime}\right), \\
& \beta_{6}=\frac{1}{4 A}\left[-4 A^{\prime}\left(r+2(1-6 B) \xi^{(1)}(\psi) \psi^{\prime}\right)+A\left(-4+r^{2} \psi^{2}\right)\right] .
\end{aligned}
$$

The coefficients in Eq. (62) are given by

$$
\begin{aligned}
\gamma_{1} & =\frac{r+2(1-3 B) \xi^{(1)}(\psi) \psi^{\prime}}{B} \\
\gamma_{2} & =-4(B-1) \xi^{(1)}(\psi) \\
\gamma_{3} & =\frac{1}{2 A}\left[4(B-1) A^{\prime} \xi^{(1)}(\psi)+A \psi^{\prime}\left(r^{2}-8(B-1) \xi^{(2)}(\psi)\right)\right] .
\end{aligned}
$$


The coefficients in Eq. (63) are given by

$$
\begin{aligned}
c_{1} & =-\frac{2 B^{\prime} \xi^{(1)}(\psi)}{A}, \\
c_{2} & =\frac{2 B^{2} A^{\prime} \xi^{(1)}(\psi)}{A}, \\
c_{3} & =\frac{B}{2 A^{2}}\left[A\left(6 A^{\prime} B^{\prime} \xi^{(1)}(\psi)-r A \psi^{\prime}\right)+B\left(-2 A^{\prime 2} \xi^{(1)}(\psi)+4 A \xi^{(1)}(\psi) A^{\prime \prime}+8 A A^{\prime} \psi^{\prime} \xi^{(2)}(\psi)\right)\right], \\
c_{4} & =\frac{B}{A^{2}}\left[3 A A^{\prime} B^{\prime} \psi^{\prime} \xi^{(2)}(\psi)+B\left(-A^{\prime 2} \psi^{\prime} \xi^{(2)}(\psi)+2 A \psi^{\prime} A^{\prime \prime} \xi^{(2)}(\psi)+2 A A^{\prime}\left(\xi^{(2)}(\psi) \psi^{\prime \prime}+\psi^{\prime 2} \xi^{(3)}(\psi)\right)\right)\right] \\
c_{5} & =-\frac{B\left(r-4 B \xi^{(1)}(\psi) \psi^{\prime}\right)}{2 A}, \\
c_{6} & =\frac{1}{4 A^{2}}\left[2 B A^{\prime}\left(r-4 B \xi^{(1)}(\psi) \psi^{\prime}\right)\right. \\
& \left.+A\left(-r B^{\prime}+2 B\left(-1+6 B^{\prime} \xi^{(1)}(\psi) \psi^{\prime}\right)+8 B^{2}\left(\psi^{\prime 2} \xi^{(2)}(\psi)+\xi^{(1)}(\psi) \psi^{\prime \prime}\right)\right)\right] \\
c_{7} & =\frac{1}{4 A^{3}}\left[2 B A^{\prime 2}\left(-r+4 B \xi^{(1)}(\psi) \psi^{\prime}\right)\right. \\
& \left.+A\left(r A^{\prime} B^{\prime}+2 B\left(A^{\prime}\left(1-6 B^{\prime} \xi^{(1)}(\psi) \psi^{\prime}\right)+r A^{\prime \prime}\right)-8 B^{2}\left(A^{\prime} \psi^{\prime 2} \xi^{(2)}(\psi)+\xi^{(1)}(\psi)\left(\psi^{\prime} A^{\prime \prime}+A^{\prime} \psi^{\prime \prime}\right)\right)\right)\right] \\
c_{8} & =-\frac{r-4 B \xi^{(1)}(\psi) \psi^{\prime}}{2 A B}, \\
c_{9} & =-\frac{1}{4 A}\left(2 A+A^{\prime}\left(r-12 B \xi^{(1)}(\psi) \psi^{\prime}\right)\right) \\
& =-\frac{1}{4 A^{2}}\left[r A^{2} \psi^{\prime 2}-A^{\prime 2}\left(r-8 B \xi^{(1)}(\psi) \psi^{\prime}\right)\right. \\
& \left.\left.=\left(r-8 B \xi^{(1)}(\psi) \psi^{\prime}\right) A^{\prime \prime}+A^{\prime}\left(-1+6 B^{\prime} \xi^{(1)}(\psi) \psi^{\prime}+8 B\left(\xi^{(2)}(\psi) \psi^{\prime 2}+\xi^{(1)}(\psi) \psi^{\prime \prime}\right)\right)\right)\right]
\end{aligned}
$$

The coefficients in Eq. (64) are given by

$$
\begin{aligned}
d_{1} & =-\frac{1}{A} \\
d_{2} & =B \\
d_{3} & =\frac{1}{2}\left(B\left(\frac{4}{r}+\frac{A^{\prime}}{A}\right)+B^{\prime}\right) \\
d_{4} & =-\frac{2 \xi^{(2)}(\psi)}{r^{2} A^{2}}\left[A A^{\prime} B^{\prime}+B^{2}\left(A^{\prime 2}-2 A A^{\prime \prime}\right)-B\left(A^{\prime 2}+3 A A^{\prime} B^{\prime}-2 A A^{\prime \prime}\right)\right] \\
d_{5} & =\frac{4(B-1) B \xi^{(1)}(\psi)}{r^{2} A} \\
d_{6} & =\frac{1}{2 r^{2} A^{2}}\left[-8 B^{2} A^{\prime} \xi^{(1)}(\psi)-4 A B^{\prime} \xi^{(1)}(\psi)+B\left(8 A^{\prime} \xi^{(1)}(\psi)+A\left(12 B^{\prime} \xi^{(1)}(\psi)+r^{2} \psi^{\prime}\right)\right)\right] \\
d_{7} & =\frac{1}{2 r^{2} A^{3}}\left[4 A A^{\prime} B^{\prime} \xi^{(1)}(\psi)+8 B^{2} \xi^{(1)}(\psi)\left(A^{\prime 2}-A A^{\prime \prime}\right)\right. \\
& \left.-B\left(8 A^{\prime 2} \xi^{(1)}(\psi)+A A^{\prime}\left(12 B^{\prime} \xi^{(1)}(\psi)+r^{2} \psi^{\prime}\right)-8 A \xi^{(1)}(\psi) A^{\prime \prime}\right)\right] \\
d_{8} & =\frac{4(B-1) \xi^{(1)}(\psi)}{r^{2} A B}, \\
d_{9} & =\frac{1}{2 r^{2} A}\left(4(3 B-1) A^{\prime} \xi^{(1)}(\psi)+r^{2} A \psi^{\prime}\right) \\
d_{10} & =\frac{1}{2 r^{2} A^{2}}\left[4(1-2 B) A^{\prime 2} \xi^{(1)}(\psi)+A A^{\prime}\left(12 B^{\prime} \xi^{(1)}(\psi)+r^{2} \psi^{\prime}\right)+2 A\left(4(2 B-1) \xi^{(1)}(\psi) A^{\prime \prime}+r A\left(2 \psi^{\prime}+r \psi^{\prime \prime}\right)\right)\right]
\end{aligned}
$$


The coefficients in Eq. (67) are given by

$$
\begin{aligned}
\rho_{1} & =-d_{5}\left(c_{1}-\frac{c_{6} \beta_{1}}{\beta_{5}}-\frac{c_{8} \gamma_{3}}{\gamma_{1}}\right)+c_{5}\left(d_{1}-\frac{d_{6} \beta_{1}}{\beta_{5}}-\frac{d_{8} \gamma_{3}}{\gamma_{1}}\right) \\
\rho_{2} & =\frac{1}{\gamma_{1}}\left(d_{5}\left(c_{2} \gamma_{1}-c_{9} \gamma_{2}\right)-c_{5}\left(d_{2} \gamma_{1}-d_{9} \gamma_{2}\right)\right) \\
\rho_{3} & =\frac{1}{\beta_{5} \gamma_{1}^{2}}\left[c_{6} d_{5} \gamma_{1}\left(-\beta_{2} \gamma_{1}+\beta_{6} \gamma_{2}\right)+d_{5} \beta_{5}\left(c_{3} \gamma_{1}^{2}-c_{10} \gamma_{1} \gamma_{2}-c_{9}\left(-\gamma_{2} \gamma_{1}^{\prime}+\gamma_{1}\left(\gamma_{3}+\gamma_{2}^{\prime}\right)\right)\right)\right. \\
& \left.-c_{5} d_{6} \gamma_{1}\left(-\beta_{2} \gamma_{1}+\beta_{6} \gamma_{2}\right)-c_{5} \beta_{5}\left(d_{3} \gamma_{1}^{2}-d_{10} \gamma_{1} \gamma_{2}-d_{9}\left(-\gamma_{2} \gamma_{1}^{\prime}+\gamma_{1}\left(\gamma_{3}+\gamma_{2}^{\prime}\right)\right)\right)\right] \\
\rho_{4} & =\frac{1}{\beta_{5} \gamma_{1}^{2}}\left[c_{6} d_{5} \gamma_{1}\left(-\beta_{3} \gamma_{1}+\beta_{6} \gamma_{3}\right)+d_{5} \beta_{5}\left(c_{4} \gamma_{1}^{2}-c_{10} \gamma_{1} \gamma_{3}+c_{9} \gamma_{3} \gamma_{1}^{\prime}-c_{9} \gamma_{1} \gamma_{3}^{\prime}\right)\right. \\
& \left.-d_{6} c_{5} \gamma_{1}\left(-\beta_{3} \gamma_{1}+\beta_{6} \gamma_{3}\right)-c_{5} \beta_{5}\left(d_{4} \gamma_{1}^{2}-d_{10} \gamma_{1} \gamma_{3}+d_{9} \gamma_{3} \gamma_{1}^{\prime}-d_{9} \gamma_{1} \gamma_{3}^{\prime}\right)\right] .
\end{aligned}
$$

[1] D. Lovelock, J. Math. Phys. 13, 874 (1972).

[2] E. Berti et al., Class. Quant. Grav. 32, 243001 (2015), arXiv:1501.07274 [gr-qc].

[3] K. Koyama, Rept. Prog. Phys. 79, 046902 (2016), arXiv:1504.04623 [astro-ph.CO].

[4] G. W. Horndeski, Int. J. Theor. Phys. 10, 363 (1974).

[5] C. Deffayet, S. Deser, and G. Esposito-Farese, Phys. Rev. D80, 064015 (2009), arXiv:0906.1967 [gr-qc].

[6] C. Deffayet, X. Gao, D. A. Steer, and G. Zahariade, Phys. Rev. D84, 064039 (2011), arXiv:1103.3260 [hep-th].

[7] T. Kobayashi, M. Yamaguchi, and J. Yokoyama, Prog. Theor. Phys. 126, 511 (2011), arXiv:1105.5723 [hep-th].

[8] J. Gleyzes, D. Langlois, F. Piazza, and F. Vernizzi, Phys. Rev. Lett. 114, 211101 (2015), arXiv:1404.6495 [hep-th].

[9] D. Langlois and K. Noui, JCAP 1602, 034 (2016), arXiv:1510.06930 [gr-qc].

[10] J. Ben Achour, M. Crisostomi, K. Koyama, D. Langlois, K. Noui, and G. Tasinato, JHEP 12, 100 (2016), arXiv:1608.08135 [hep-th].

[11] L. Barack et al., (2018), arXiv:1806.05195 [gr-qc].

[12] W. Israel, Phys. Rev. 164, 1776 (1967).

[13] B. Carter, Phys. Rev. Lett. 26, 331 (1971).

[14] R. Ruffini and J. A. Wheeler, Phys. Today 24, 30 (1971).

[15] S. W. Hawking, Commun. Math. Phys. 25, 152 (1972).

[16] J. E. Chase, Commun. Math. Phys. 19, 276 (1970).

[17] S. W. Hawking, Commun. Math. Phys. 25, 167 (1972).

[18] J. D. Bekenstein, Phys. Rev. D51, R6608 (1995).

[19] A. A. H. Graham and R. Jha, Phys. Rev. D89, 084056 (2014), [Erratum: Phys. Rev.D92,no.6,069901(2015)], arXiv:1401.8203 [gr-qc].

[20] A. A. H. Graham and R. Jha, Phys. Rev. D90, 041501 (2014), arXiv:1407.6573 [gr-qc].

[21] C. A. R. Herdeiro and E. Radu, Proceedings, 7th Black Holes Workshop 2014: Aveiro, Portugal, December 18-19, 2014, Int. J. Mod. Phys. D24, 1542014 (2015), arXiv:1504.08209 [gr-qc].

[22] L. Hui and A. Nicolis, Phys. Rev. Lett. 110, 241104 (2013), arXiv:1202.1296 [hep-th].

[23] E. Babichev, C. Charmousis, and A. Lehebel, JCAP 1704, 027 (2017), arXiv:1702.01938 [gr-qc].

[24] E. Barausse and T. P. Sotiriou, Phys. Rev. Lett. 101, 099001 (2008), arXiv:0803.3433 [gr-qc].

[25] O. J. Tattersall and P. G. Ferreira, Phys. Rev. D97, 104047 (2018), arXiv:1804.08950 [gr-qc].

[26] P. Kanti, N. E. Mavromatos, J. Rizos, K. Tamvakis, and E. Winstanley, Phys. Rev. D54, 5049 (1996), arXiv:hep-th/9511071 [hep-th].

[27] S. O. Alexeev and M. V. Pomazanov, Phys. Rev. D55, 2110 (1997), arXiv:hep-th/9605106 [hep-th].

[28] T. Torii, H. Yajima, and K.-i. Maeda, Phys. Rev. D55, 739 (1997), arXiv:gr-qc/9606034 [gr-qc].

[29] P. Kanti, N. E. Mavromatos, J. Rizos, K. Tamvakis, and E. Winstanley, Phys. Rev. D57, 6255 (1998), arXiv:hep-th/9703192 [hep-th].

[30] C.-M. Chen, D. V. Gal'tsov, and D. G. Orlov, Phys. Rev. D75, 084030 (2007), arXiv:hep-th/0701004 [hep-th].

[31] Z.-K. Guo, N. Ohta, and T. Torii, Prog. Theor. Phys. 120, 581 (2008), arXiv:0806.2481 [gr-qc].

[32] Z.-K. Guo, N. Ohta, and T. Torii, Prog. Theor. Phys. 121, 253 (2009), arXiv:0811.3068 [gr-qc].

[33] N. Ohta and T. Torii, Prog. Theor. Phys. 121, 959 (2009), arXiv:0902.4072 [hep-th].

[34] N. Ohta and T. Torii, Prog. Theor. Phys. 122, 1477 (2009), arXiv:0908.3918 [hep-th].

[35] B.-H. Lee, W. Lee, and D. Ro, Phys. Rev. D99, 024002 (2019), arXiv:1809.05653 [gr-qc].

[36] P. Pani and V. Cardoso, Phys. Rev. D79, 084031 (2009), arXiv:0902.1569 [gr-qc].

[37] D. Ayzenberg and N. Yunes, Phys. Rev. D90, 044066 (2014), [Erratum: Phys. Rev.D91,no.6,069905(2015)], arXiv:1405.2133 [gr-qc].

[38] A. Maselli, P. Pani, L. Gualtieri, and V. Ferrari, Phys. Rev. D92, 083014 (2015), arXiv:1507.00680 [gr-qc]. 
[39] B. Kleihaus, J. Kunz, and E. Radu, Phys. Rev. Lett. 106, 151104 (2011), arXiv:1101.2868 [gr-qc].

[40] B. Kleihaus, J. Kunz, S. Mojica, and E. Radu, Phys. Rev. D93, 044047 (2016), arXiv:1511.05513 [gr-qc].

[41] T. P. Sotiriou and S.-Y. Zhou, Phys. Rev. Lett. 112, 251102 (2014), arXiv:1312.3622 [gr-qc].

[42] T. P. Sotiriou and S.-Y. Zhou, Phys. Rev. D90, 124063 (2014), arXiv:1408.1698 [gr-qc].

[43] T. Damour and G. Esposito-Farese, Phys. Rev. Lett. 70, 2220 (1993).

[44] T. Damour and G. Esposito-Farese, Phys. Rev. D54, 1474 (1996), arXiv:gr-qc/9602056 [gr-qc].

[45] J. Novak, Phys. Rev. D57, 4789 (1998), arXiv:gr-qc/9707041 [gr-qc].

[46] T. Harada, Phys. Rev. D57, 4802 (1998), arXiv:gr-qc/9801049 [gr-qc].

[47] P. C. C. Freire, N. Wex, G. Esposito-Farese, J. P. W. Verbiest, M. Bailes, B. A. Jacoby, M. Kramer, I. H. Stairs, J. Antoniadis, and G. H. Janssen, Mon. Not. Roy. Astron. Soc. 423, 3328 (2012), arXiv:1205.1450 [astro-ph.GA].

[48] H. O. Silva, J. Sakstein, L. Gualtieri, T. P. Sotiriou, and E. Berti, Phys. Rev. Lett. 120, 131104 (2018), arXiv:1711.02080 [gr-qc].

[49] D. D. Doneva and S. S. Yazadjiev, Phys. Rev. Lett. 120, 131103 (2018), arXiv:1711.01187 [gr-qc].

[50] G. Antoniou, A. Bakopoulos, and P. Kanti, Phys. Rev. Lett. 120, 131102 (2018), arXiv:1711.03390 [hep-th].

[51] G. Antoniou, A. Bakopoulos, and P. Kanti, Phys. Rev. D97, 084037 (2018), arXiv:1711.07431 [hep-th].

[52] D. D. Doneva and S. S. Yazadjiev, JCAP 1804, 011 (2018), arXiv:1712.03715 [gr-qc].

[53] J. L. Blzquez-Salcedo, D. D. Doneva, J. Kunz, and S. S. Yazadjiev, Phys. Rev. D98, 084011 (2018), arXiv:1805.05755 [gr-qc].

[54] D. D. Doneva, S. Kiorpelidi, P. G. Nedkova, E. Papantonopoulos, and S. S. Yazadjiev, (2018), arXiv:1809.00844 [gr-qc].

[55] B. Carr, F. Kuhnel, and M. Sandstad, Phys. Rev. D94, 083504 (2016), arXiv:1607.06077 [astro-ph.CO].

[56] C. A. R. Herdeiro, E. Radu, N. Sanchis-Gual, and J. A. Font, Phys. Rev. Lett. 121, 101102 (2018), arXiv:1806.05190 [gr-qc].

[57] I. Z. Stefanov, S. S. Yazadjiev, and M. D. Todorov, Mod. Phys. Lett. A23, 2915 (2008), arXiv:0708.4141 [gr-qc].

[58] D. D. Doneva, S. S. Yazadjiev, K. D. Kokkotas, and I. Z. Stefanov, Phys. Rev. D82, 064030 (2010), arXiv:1007.1767 [gr-qc]. 